\title{
Study of Einstein-bumblebee gravity with Kerr-Sen-like solution in the presence of a dispersive medium
}

\author{
Sohan Kumar Jha ${ }^{1}$, Sahazada Aziz ${ }^{2}$, Anisur Rahaman ${ }^{3, \text { a }}$ \\ ${ }^{1}$ Chandernagore College, Chandernagore, Hooghly, West Bengal 712136, India \\ ${ }^{2}$ Ramananda Centenary College, Laulara, Purulia, West Bengal 723151, India \\ ${ }^{3}$ Durgapur Govt. College, Durgapur, Burdwan, West Bengal 713214, India
}

Received: 12 October 2021 / Accepted: 19 January 2022 / Published online: 3 February 2022

(C) The Author(s) 2022

\begin{abstract}
A Kerr-Sen-like black hole solution appears in the Einstein-bumblebee theory of gravity. The solution contains contains a Lorentz violating parameter in an explicit manner. We study the null geodesics in the background of this Kerr-Sen-like black hole surrounded by a dispersive medium like plasma. We investigate the effect of the charge of the black hole, the Lorentz violation parameter, and the plasma parameter on the photon orbits with the evaluation of the effective potential in the presence of both the Lorentz violation parameter and the plasma parameter. We also study the influence of the Lorentz violation parameter and plasma parameter on the emission of energy from the black hole due to thermal radiation. Besides, we compute the angle of deflection of massless particles with weak-field approximation in this generalized situation and examine how it varies with the Lorentz violation parameter in presence of plasma. Constraining the parameters of this Lorentz violating KerrSen-like black hole is also attempted here with the result obtained from the observations of the Event Horizon Telescope (EHT) collaboration.
\end{abstract}

\section{Introduction}

The physics of black holes has got renewed interest after the announcement of the detection of gravitational waves (GWs) [1] by the LIGO and VIRGO observatories and the captured image of the black hole shadow of a super-massive M87* black hole by the EHT based on the very long baseline interferometry (VIBI) [2-7]. The shadow of black-hole is well studied from the theory of General relativity. A black hole shadow is a two-dimensional dark zone in the celestial sphere caused by the strong gravity of the black hole. In the

\footnotetext{
a e-mails: anisur.associates@ iucaa.ac.in; manisurn@ gmail.com (corresponding author)
}

article [8], Synge studied the shadow of the Schwarzschild black hole, which was then termed as the escape cone of light. The radius of the shadow of the black hole was calculated in terms of the mass of the black hole and the radial coordinate where the observer is located. In general, the shadow of a nonrotating black hole is a standard circle, whereas it is known that the shadow of a rotating black hole is not a circular disk. The different features of the black hole have been widely investigated for various gravity backgrounds, adopting an almost similar approach based on classical dynamics [9-26].

In General Relativity, light is attributed to the null geodesic of the spacetime metric and the in-medium effect, which is negligible for most of the frequency ranges, is significant for the radio frequency range. In this context, it is worth mentioning the impact of Solar corona on the time of travel and on the deflection angle of radio signals that come close to the Sun. Since the 1960s, this influence has been routinely observed. In this case, one may assume that the medium is a non-magnetized pressureless plasma and for the gravitational field, the linearized theory is sufficient. The relevant theoretical development has been made by Muhleman et al. in the articles $[27,28]$. With the available information, the shadow of black holes in presence of plasma has become an interesting field of research for physicists since there is a good reason for considering that black holes and other compact objects are surrounded by plasma. So, naturally, interest grows to investigate whether the presence of plasma leads to any observational effect on the radio signals and several investigations have been carried out in that direction [29-40]. The physics of black holes is crucially linked with the Planck energy scale where gravity is sufficiently strong and a classical field theory of curved spacetime is not adequate to capture the finer details of the Planck scale. It needs quantization of gravity, which is not well-developed. However, it is reasonable to accept that the nature of the spacetime is discrete in that scale. Lorentz symmetry being a continuous symme- 
try, would not be tenable in discrete spacetime. Moreover, according to string theory, Lorentz invariance should not be an exact principle at all energy scales [41-44]. If Lorentz violation (LV) is considered to be a probe for the foundation of theoretical physics, then a suitable model is needed, which contains LV adopted from the standard physical principle. The known two well-developed and successful theories that describe our Universe are the theory of General Relativity and the Standard Model of particle physics. These theories are built up obeying Lorentz invariance and these two theories are applicable in two different energy scales. The standard model describes the three fundamental interactions at the quantum level, whereas general relativity describes the gravitational interaction at the classical level. A single complete theory requires the unification of these two. However, there is a strong obstacle in the way of unification since gravity can not be quantized in a straightforward manner. Therefore, a successful unified theory has not yet been developed even with huge efforts from different fronts. To unify them at very high energy scales, one builds an effective field theory termed as Standard Model Extension (SME), that couples the Standard Model to the theory of General Relativity, which involves extra items containing information about the LV happening at the Plank scale [45-47]. The simplest case is described by a single vector bumblebee field with a nonzero vacuum expectation value and the spontaneous LV triggered by a smooth quadratic potential. Bumblebee gravitational model was first studied by Kostelecky and Samuel in [4143] as a specific pattern for unprompted Lorentz violation. Therefore, the Lorentz violation aspect may be considered as a good probe on the foundation of physics.

In this context, the study of the LV effect on the shadow of a black hole in the presence of dispersive media like plasma would be of interest. There are several recent investigations to study the deformation of black hole shadow due to the LV effect [48-50]. In these studies, the in-medium (plasma) effect has not been considered so far. Therefore, the study of the deformation of the shadow of the black hole connected with Kerr-Sen-like spacetime, the emission of energy from this black hole due to radiation, and the weak lensing in the presence of plasma would be interesting extensions. The black hole associated with Kerr-Sen-like spacetime [50] contains an LV parameter as both the Swarczschild-like and Kerrlike spacetime contain $[48,49]$. This Kerr-Sen-like spacetime will enable us to study the LV effect in presence of plasma.

It is known that observation of the EHT collaboration has unveiled the first shadow image of a supermassive black hole M87* with an asymmetric bright emission ring with a diameter of $42 \pm 3 \mu$ as exhibiting a deviation from circularity $\Delta C \leq 0.10$. It is consistent with the shadow of the Kerr black hole, but the quantitative features are not always sufficient to distinguish between black holes predicted from different theories of gravity. Thus, there is enough room for investigation concerning the viability of black holes predicted from modified theories of gravity with the help of the observed data of M87* black hole shadow by the EHT collaboration placing constraints on the black hole parameters, and consequently, it becomes an important tool to test the phenomena of strong gravitational fields. Extensive development in this direction is going on [52-58].

Therefore, in this paper, we will consider Einstein's gravity, which is coupled to the bumblebee field to get some suppressed effects emerging from the underlying unified quantum gravity theory on our low energy scale. Recently, Casana et al. [48] gave an exact Schwarzschild-like solution of this bumblebee gravity model, and considered some classical tests. The rotating black hole solutions are the most relational subsets of astrophysics. In [49], Ding et al. found an exact Kerr-like solution by solving Einstein-bumblebee gravitational field equations and studied its black hole shadow. Later on, in the article [59], the authors have studied the weak gravitational deflection angle of relativistic massive particles by this Kerr-like black hole. In [50], we have presented a Kerr-Sen-like modified solution. It has an LV parameter and the presence of plasma supplies an additional parameter. In this article along with the study of theoretical aspects concerning the Kerr-Sen-like black hole in the presence of plasma, we have made an attempt to constrain the parameters of Kerr-Sen-like modified black holes considering M87* as Kerr-Sen-black holes using the recently obtained result of the EHT collaboration.

The article is organized as follows. In Sect. 2, we study the null geodesics around the charged rotating Kerr-Sen-like black hole in the presence of plasma. In Sect. 3, we study the shadow corresponding to this charged rotating black hole and how the shadow gets deformed with the variation of charge, LV parameter, and the plasma parameter. Section 4 is devoted to the study of the emission of energy due to thermal radiation for this black hole, and the study of the effect of the LV parameter and the plasma parameter on it. In Sect. 5, we study the deflection angle of light with weak-field approximation in this LV spacetime background in the presence of plasma. Section 6 is devoted to constraining the parameters of the Kerr-Sen-like black hole with the experimentally observed quantities for the M87* black hole. The final Sect. 7 contains a summary and discussion.

\section{Null geodesics around the charged rotating Kerr-Sen-like black hole in the presence of plasma}

It is known that Einstein-bumblebee gravity model is a modification over the Einstein's gravity where a pseudo vector field namely bumblebee field was introduced that broke Lorentz symmetry, acquiring a vacuum expectation value. In the article [48], the authors showed that this modified 
theory provided for a Schwarzschild-like solution. A Kerrlike solution was developed in [49]. We have shown that Einstein-bumblebee gravity model also renders a Kerr-Senlike black hole metric. It is the outcome of the introduction of the Bumblebee field in the Kerr-Sen background [51] In Boyer-Lindquist coordinates, the Kerr-Sen-like charged rotating metric that results out from the Einstein-bumblebee gravity reads [50]

$$
\begin{aligned}
d s^{2}= & -\left(1-\frac{2 M r}{\rho^{2}}\right) d t^{2} \\
& -\frac{4 M r a \sqrt{1+l} \sin ^{2} \theta}{\rho^{2}} d t d \varphi+\frac{\rho^{2}}{\Delta} d r^{2} \\
& +\rho^{2} d \theta^{2}+\frac{A \sin ^{2} \theta}{\rho^{2}} d \varphi^{2},
\end{aligned}
$$

where

$$
\begin{aligned}
\rho^{2} & =r(r+b)+a^{2}(1+l), \Delta=\frac{r(r+b)-2 M r}{1+l}+a^{2}, \\
A & =\left[r(r+b)+(1+l) a^{2}\right]^{2}-\Delta(1+l)^{2} a^{2} \sin ^{2} \theta .
\end{aligned}
$$

The parameters $a$ and $b$ are related with angular momentum and the charge of the black hole by the relations $a=\frac{J}{M}$ the $Q=\sqrt{b M}$. Here $M, Q$ and $J$ respectively refer to the mass, charge and angular momentum. The expression of $\rho$ and $\Delta$ contain the parameter $l$, that represents the Lorentz violation parameter, which is associated with spontaneous violation of symmetry of the vacuum of the bumblebee field. If $l \rightarrow 0$ it recovers the usual Kerr-Sen metric and for $a \rightarrow 0$ and $b \rightarrow 0$ the metric turns into

$$
\begin{aligned}
d s^{2}= & -\left(1-\frac{2 M}{r}\right) d t^{2}+\frac{1+l}{1-2 M / r} d r^{2} \\
& +r^{2} d \theta^{2}+r^{2} \sin ^{2} \theta d \varphi^{2},
\end{aligned}
$$

which is the Schwarzschild-like solution for the Einsteinbumblebee gravity $[48,49]$.

We consider a static inhomogeneous plasma in the gravitational field with a refractive index $n$. The expression of refractive index $n$, as formulated by Synge [60], in terms of dynamical variables reads

$n^{2}=1+\frac{p_{\mu} p^{\mu}}{\left(p_{\nu} u^{\nu}\right)^{2}}$,

where $p_{\mu}$ and $u^{v}$ are four-momentum and four-velocity of the massless particle respectively. Let us now start with the Hamiltonian for massless particles like photon:

$H\left(x^{\mu}, p_{\mu}\right)=\frac{1}{2}\left[g^{\mu \nu} p_{\mu} p_{\nu}-\left(n^{2}-1\right)\left(p_{0} \sqrt{-g^{00}}\right)^{2}\right]$.

The standard definitions $x^{\mu}=\partial H / \partial p_{\mu}$, and $\dot{p_{\mu}}=\partial H / \partial x^{\mu}$ lead us to write down the equations of motion for photon in the plasma medium as follows

$$
\begin{aligned}
\rho^{2} \frac{d r}{d \lambda} & = \pm \sqrt{R}, \quad \rho^{2} \frac{d \theta}{d \lambda}= \pm \sqrt{\Theta}, \\
(1+l) \Delta \rho^{2} \frac{d t}{d \lambda} & =A n^{2}-2 \sqrt{1+l} M r a \xi \\
(1+l) \Delta \rho^{2} \frac{d \phi}{d \lambda} & =2 M r a \sqrt{1+l}+\frac{\xi}{\sin ^{2} \theta}\left(\rho^{2}-2 M r\right),
\end{aligned}
$$

where $\lambda$ is the affine parameter and

$$
\begin{aligned}
R(r)= & {\left[\frac{r(r+b)+(1+l) a^{2}}{\sqrt{1+l}}-a \xi\right]^{2} } \\
& -\Delta\left[\eta+(\xi-\sqrt{1+l} a)^{2}\right] \\
& +\left(n^{2}-1\right) \frac{\left[r(r+b)+a^{2}(1+l)\right]^{2}}{1+l}, \\
\Theta(\theta)= & \eta+(1+l) a^{2} \cos ^{2} \theta-\xi^{2} \cot ^{2} \theta \\
& -\left(n^{2}-1\right) a^{2}(1+l) \sin ^{2} \theta .
\end{aligned}
$$

In Eq. (9), we introduce two conserved parameters $\xi$ and $\eta$ as usual, which are defined by

$\xi=\frac{L_{z}}{E} \quad$ and $\quad \eta=\frac{\mathcal{Q}}{E^{2}}$,

where $E, L_{z}$ and $\mathcal{Q}$ are the energy, the axial component of the angular momentum, and the Carter constant respectively.

We introduce a static inhomogeneous plasma with a refractive index $n$, which depends on the space location $x$, and the photon frequency $\omega$ [38]. In terms of $\omega$, the square of the refractive index $n$ is defined by

$n^{2}=1-\frac{\omega_{e}^{2}}{\omega^{2}}$

where $\omega$ and $\omega_{e}$ are, respectively, the frequency of photon and the electron-plasma frequency. In the general theory of relativity, the redshift scenario entails that frequency of photons depends on the spatial coordinates due to the presence of the gravitational field. Besides, the electron-plasma frequency has the expression [61]

$\omega_{e}^{2}=\frac{4 \pi e^{2} N(r)}{m}$,

where $N(r)$ is the concentration of electron in the inhomogeneous plasma. The mass and the charge of the electron are respectively denoted by $m$ and $e$. We now consider a radial power-law density as

$N(r)=\frac{N_{0} r_{0}}{r^{h}}$,

where $N_{0}$ is the density number at the radial position of the inner edge of plasma environment $r_{0}$. Therefore, we have

$\omega_{e}^{2}=\frac{4 \pi e^{2} N_{0} r_{0}}{m r^{h}}$, 
where $h \geq 0$. Here we consider $h=1$, as it has been considered in the article [62], so that we have

$n=\sqrt{1-\frac{k}{r}}$.

With this specific form of refractive index, we study various aspects of photon geodesics with the spacetime metric given in Eq. (1). The radial equation of motion has the known form

$$
\left(\rho^{2} \frac{d r}{d \lambda}\right)^{2}+V_{e f f}=0
$$

The effective potential $V_{e f f}$ in this situation reads

$$
\begin{aligned}
V_{e f f}= & -\left[\frac{r(r+b)+(1+l) a^{2}}{\sqrt{1+l}}-a \xi\right]^{2} \\
& +\Delta\left[\eta+(\xi-\sqrt{1+l} a)^{2}\right]-\left(n^{2}-1\right) \\
& \times \frac{\left[r(r+b)+a^{2}(1+l)\right]^{2}}{1+l} .
\end{aligned}
$$

Note that it contains both factors $l$ and $k$. The shape of the orbit crucially depends on the nature of the effective potential. Therefore, it is natural that the orbit will have crucial dependence on both factors $l$ and $k$. The unstable spherical orbit on the equatorial plane will be obtained if the following conditions are met:

$$
\begin{aligned}
& \theta=\frac{\pi}{2}, \quad R(r)=0, \quad \frac{d R}{d r}=0, \\
& \frac{d^{2} R}{d r^{2}}>0, \quad \eta=0 .
\end{aligned}
$$

We plot $V_{\text {eff }}$ versus $r / M$ with $\xi=\xi_{c}+0.2$, where $\xi_{c}$ is the value of $\xi$ for equatorial spherical unstable direct orbit. In Fig. 1, we find that the turning point moves to the left with the increase of the value of $b$ for fixed values of $k$ and $l$. It also shifts to the left with the increasing value of $k$ when $b$ and $l$ remain fixed. For the positive value of the Lorentz violating parameter $l$, it also shifts to the left and for the negative value of the $l$ it gets shifted towards the right like the case when plasma was absent [50]. In Fig. 2, if we observe the plot of the critical radius we find that $r_{c}$ decreases with the increase of the value of $b$ for fixed $k$ and $l$; and it increases with the increase of the value of $k$ when $b$ and $l$ remain fixed. However, for $l>0$ the critical radius $r_{c}$ decreases and for $l<0$ it increases.

We also plot critical radius versus $a$ keeping $\theta=\frac{\pi}{2}$. To plot it, we consider variation of the parameters $b, k$ and $l$ involved in our proposed model.

For more generic spherical orbits where $\theta \neq \pi / 2$ and $\eta \neq 0$, the conserved quantities $\xi_{s}$ and $\eta_{s}$ for $r=r_{s}$, are given by the simultaneous solutions of the equations

$R=0$ and $\frac{d R}{d r}=0$. where

$$
\begin{aligned}
R(r)= & {\left[\frac{r(r+b)+(1+l) a^{2}}{\sqrt{1+l}}-a \xi\right]^{2} } \\
& -\Delta\left[\eta+(\xi-\sqrt{1+l} a)^{2}\right] \\
& +\left(n^{2}-1\right) \frac{\left[r(r+b)+a^{2}(1+l)\right]^{2}}{1+l}
\end{aligned}
$$

and

$$
\begin{aligned}
\frac{d R}{d r}= & 2 \frac{2 r+b}{\sqrt{1+l}}\left[\frac{r(r+b)+(1+l) a^{2}}{\sqrt{1+l}}-a \xi\right] \\
& -\frac{2 r+b-2 M}{1+l}\left[\eta+(\xi-\sqrt{1+l} a)^{2}\right] \\
& +\frac{k}{r^{2}} \frac{\left[r(r+b)+a^{2}(1+l)\right]^{2}}{1+l} \\
& -2 \frac{k}{r(1+l)}(2 r+b)\left[r(r+b)+a^{2}(1+l)\right]
\end{aligned}
$$

The conserved quantities $\xi_{s}$ and $\eta_{s}$ are obtained by solving the equations

$\left.R\right|_{r=r_{s}}=0$ and $\left.\frac{d R}{d r}\right|_{r=r_{s}}=0$,

which give

$$
\begin{aligned}
& \xi_{s}=-\frac{1}{a^{2} \sqrt{1+l}(b-2 M+2 r)} \\
& \times\left(2 a^{3} M+2 a^{3} l M-2 a M r^{2}+\sqrt{1+l}\right. \\
& \times\left(\frac { a ^ { 2 } } { ( 1 + l ) r ^ { 2 } } \left(4 M^{2} r^{2}\left(-a^{2}(1+l)+r^{2}\right)^{2}\right.\right. \\
& +(b-2 M+2 r)\left(a^{2}(1+l)+r(b+r)\right) \\
& \times\left(a^{4} k(1+l)^{2}+r^{3}\left(b^{2}+4 k M-b(k+2 M-3 r)\right.\right. \\
& \left.-(k+6 M) r+2 r^{2}\right)+a^{2}(1+l) r \\
& \left.\times(-4 k M+b(k+r)+2 r(M+r)))))^{1 / 2}\right) \\
& \eta_{s}=\frac{1}{a^{3}(1+l)^{3 / 2} r(b-2 M+2 r)^{2}} \\
& \times\left(-a^{5} k(1+l)^{5 / 2}(b-2 M+2 r)(2 b-4 M+3 r)\right. \\
& -2 a^{3}(1+l)^{3 / 2} r\left(b k(b-2 M)^{2}+4 b k(b-2 M) r\right. \\
& \left.+(5 b k-2(b+k) M) r^{2}+2(k-2 M) r^{3}\right) \\
& -a r^{3} \sqrt{1+l}\left(b(b-2 M)\left(b^{2}+4 k M-b(k+2 M)\right)\right. \\
& +2\left(3 b^{3}-4 k M^{2}+8 b M(k+M)-2 b^{2}(k+5 M)\right) r \\
& +\left(13 b^{2}+10 M(k+2 M)-b(5 k+32 M)\right) \\
& \left.r^{2}+2(6 b-k-8 M) r^{3}+4 r^{4}\right) \\
& +4(1+l) M r^{3}\left(\frac{a^{8} k(1+l)^{2}(b-2 M+2 r)}{r^{2}}\right. \\
& +\frac{a^{6}(1+l)\left(2 k(b-2 M)^{2}+\left(b^{2}+5 b k-10 k M\right) r+2(2 b+k) r^{2}+4 r^{3}\right)}{r}
\end{aligned}
$$




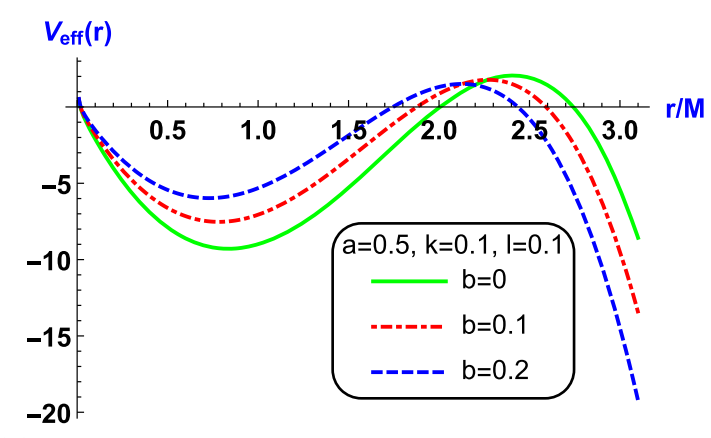

(a) Variation of the effective potential $\left(V_{\text {eff }}\right)$ with respect to $r / M$ for various values of $b$ with $a=0.5, l=0.1, k=0.1$, and $\theta=\pi / 2$.

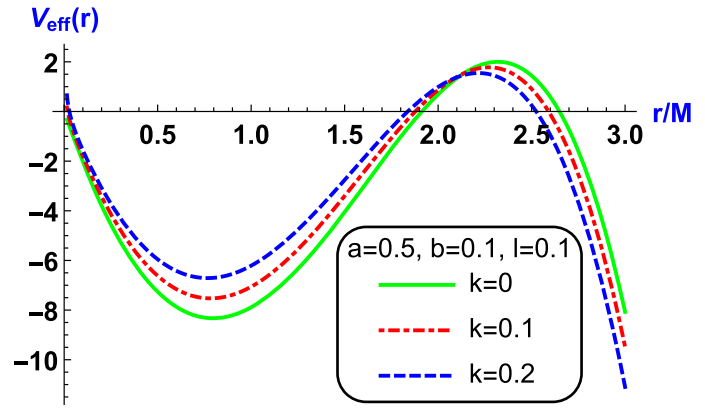

(b) Variation of the effective potential $\left(V_{e f f}\right)$ with respect to $r / M$ for various values of $k$ with $a / M=0.5, l=0.1, b=0.1$, and $\theta=\pi / 2$.

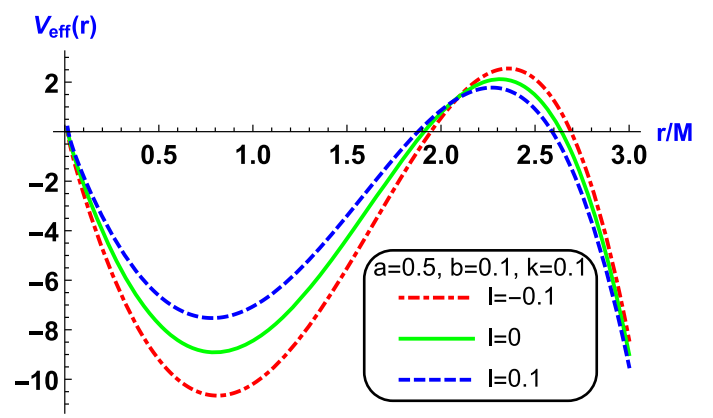

(c) Variation of effective potential $\left(V_{e f f}\right)$ with respect to

$r / M$ for various values of $l$ with $a / M=0.5, k=0.1, b=0.1$, and $\theta=\pi / 2$.

Fig. 1 Plots of the effective potential for various scenarios

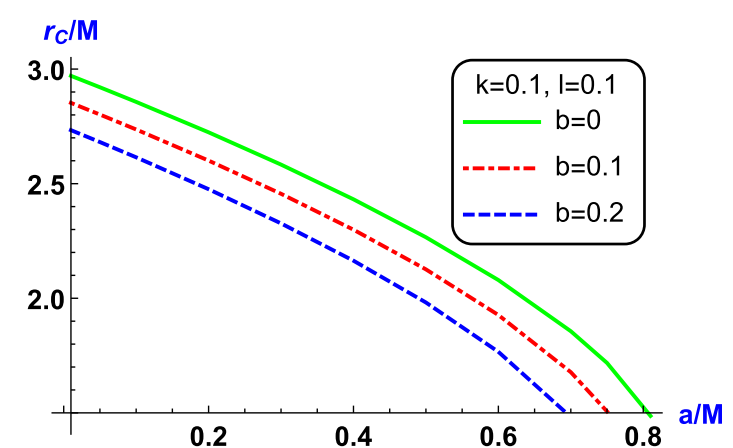

(a) Variation of the critical radii $r_{c}$ with respect to $a / M$ for various values of $b$ with $l=0.1, k=0.1$, and $\theta=\pi / 2$.

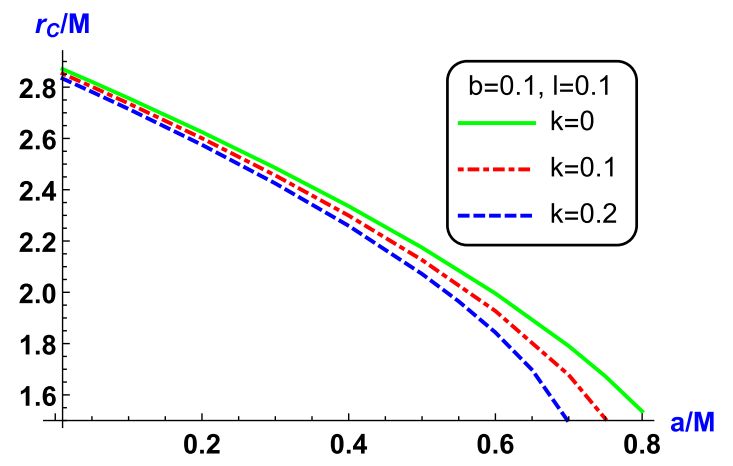

(b) Variation of the critical radii $r_{c}$ with respect to $a / M$ for various values of $k$ with $l=0.1, b=0.1$, and $\theta=\pi / 2$.

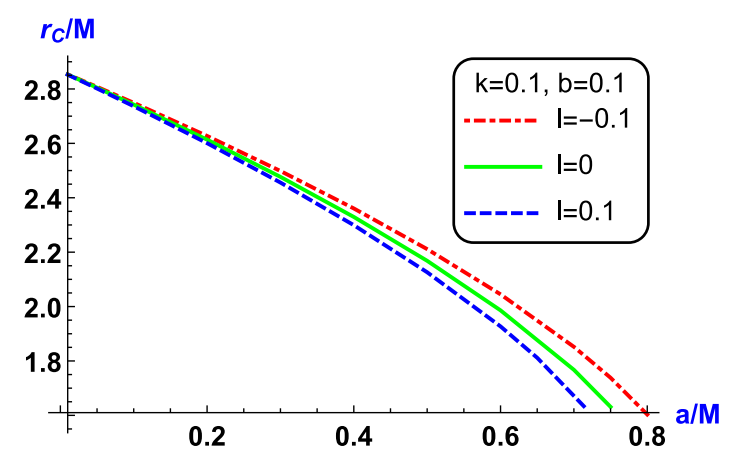

(c) Variation of the critical radii $r_{c}$ with respect to $a / M$ for various values of $l$ with $k=0.1, b=0.1$, and $\theta=\pi / 2$.

Fig. 2 Plots of critical radius for various scenarios 

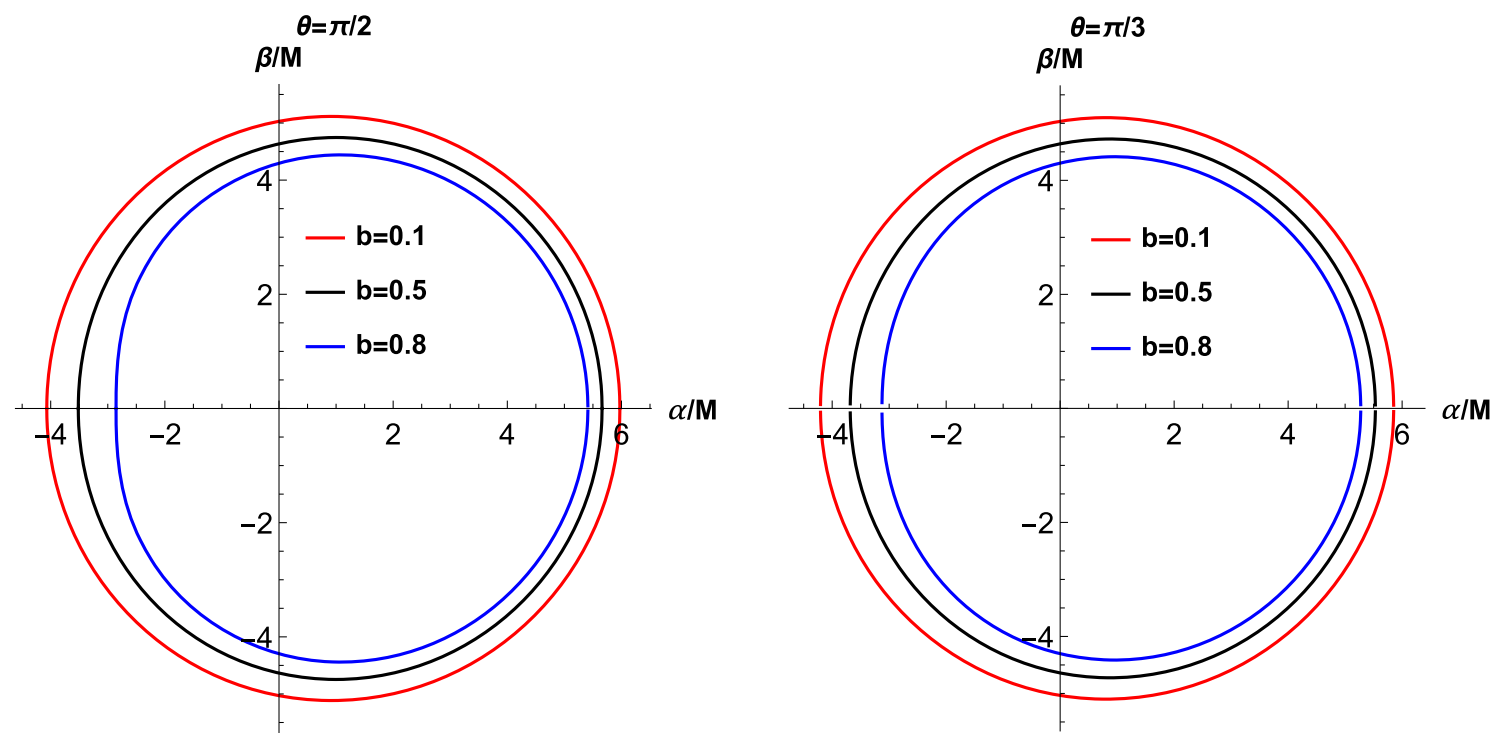

Fig. 3 The figure of the shadows for different values of $b$ when $\theta=\frac{\pi}{2}$ and $\theta=\frac{\pi}{3}$ with $a=0.4, k=0.2$, and $l=0.2$
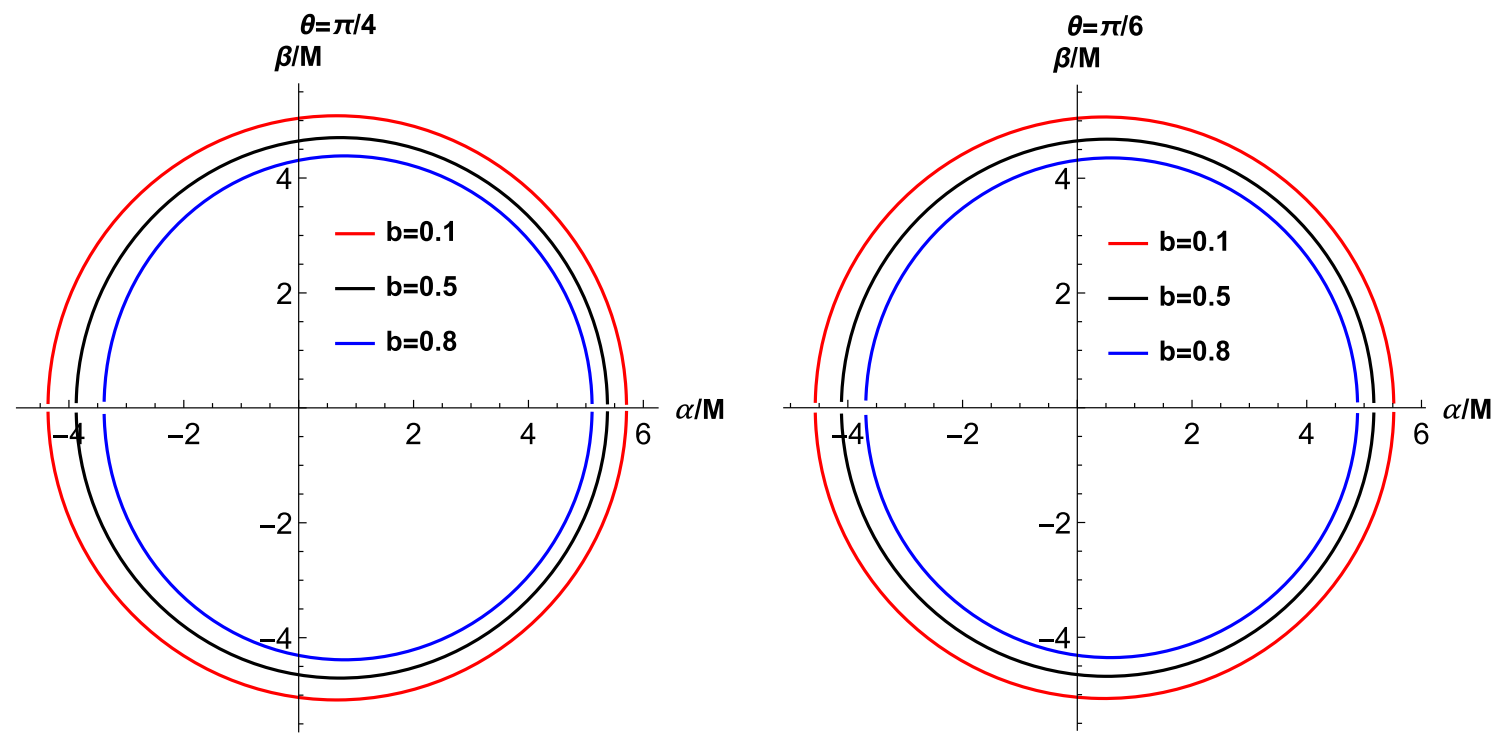

Fig. 4 The figure of the shadows for different values of $b$ when $\theta=\frac{\pi}{4}$ and $\theta=\frac{\pi}{6}$ with $a=0.4, k=0.2$, and $l=0.2$

$$
\begin{aligned}
& +\frac{a^{2} r^{2}}{1+l}\left(b(b-2 M)\left(b^{2}+4 k M-b(k+2 M)\right)\right. \\
& +2\left(3 b^{3}-4 k M^{2}+8 b M(k+M)-2 b^{2}(k+5 M)\right) r \\
& +\left(13 b^{2}+2 M(5 k+8 M)-b(5 k+32 M)\right) \\
& \left.\times r^{2}+2(6 b-k-8 M) r^{3}+4 r^{4}\right)+a^{4}(b k(b-4 M)(b-2 M) \\
& +2 b(b(b+k)-2(b+2 k) M) r \\
& +\left(10 b^{2}+2 k M-b(k+16 M)\right) \\
& \left.\left.r^{2}+2(8 b-k-8 M) r^{3}+8 r^{4}\right)\right)
\end{aligned}
$$

\section{Black hole shadow}

To describe the nature of the shadow, that an observer see in the sky, the following two celestial coordinates will be helpful

$\alpha(\xi, \eta ; \theta)=-\frac{\xi_{s} \csc \theta}{n}$,

$\beta(\xi, \eta ; \theta)$

$$
=\frac{\sqrt{\eta+(1+l) a^{2} \cos ^{2} \theta-\xi^{2} \cot ^{2} \theta-\left(n^{2}-1\right) a^{2}(1+l) \sin ^{2} \theta}}{n} .
$$



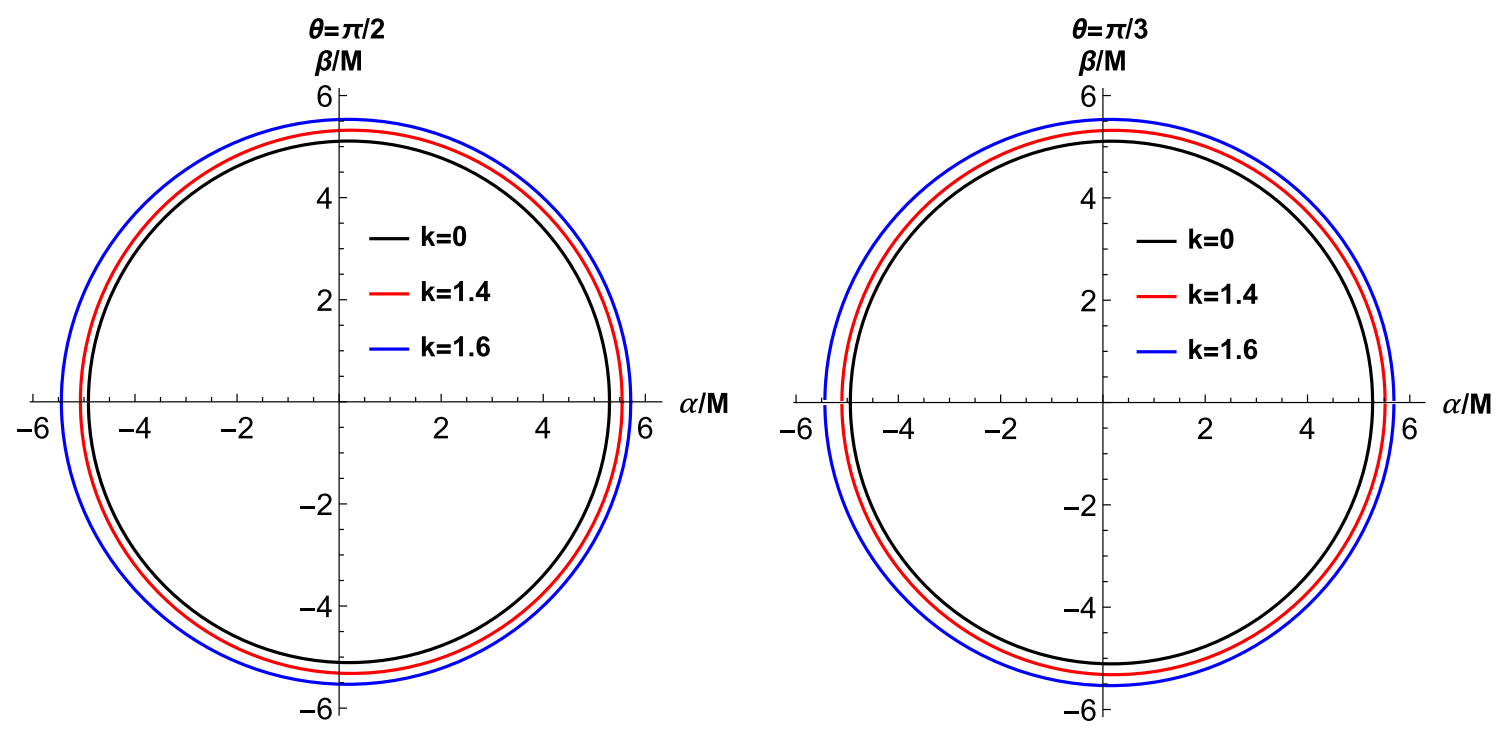

Fig. 5 The figure of the shadows for different values of $k$ when $\theta=\frac{\pi}{2}$ and $\theta=\frac{\pi}{3}$ with $a=0.1, b=0.1$, and $l=-0.1$
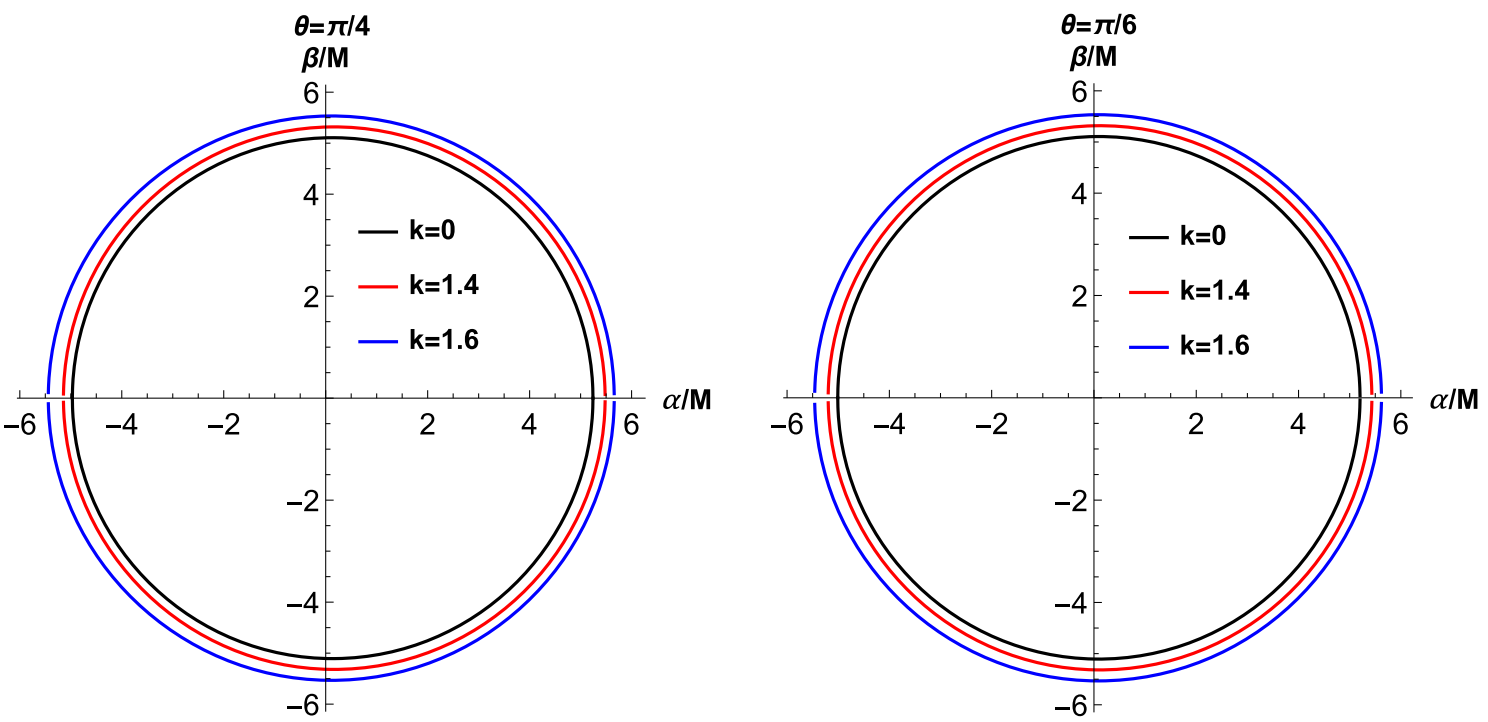

Fig. 6 The figure of the shadows for different values of $k$ when $\theta=\frac{\pi}{4}$ and $\theta=\frac{\pi}{6}$ with $a=0.1, b=0.1$, and $l=-0.1$

Let us now proceed to see the shape and size of the shadows. The nature of the shadow will depend on various parameters. So, we sketch the black hole shadow for various possible cases. In Figs. 3 and 4 we see that with the increase in the value of $b$ the size of the shadow decreases and the left end of the shadow moves a little to the right when $k$ and $l$ remain fixed. Figures 5 and 6 show the change of shape of the shadow with a variation of the plasma parameter $k$ keeping $b$ and $l$ fixed. The figure shows that the size of the shadow increases with the increase of the value of $k$. Here deformation of the shape of the shadow can not be found pictorially however mathematically very little deviation is observed, which can be observed from Table 1. Figures 7 and 8 show that for a negative value of $l$ the left end of the shadow shifts towards
Table 1 Deviation $\delta_{s}$ for Kerr-Sen-like black hole with $a=0.2$ and $b=0.2$

\begin{tabular}{llll}
\hline & $l=-0.2$ & $l=0$ & $l=0.2$ \\
\hline$k=0$ & 0.00444613 & 0.00557717 & 0.00671623 \\
$k=0.01$ & 0.00452873 & 0.00568118 & 0.00684196 \\
$k=0.02$ & 0.00461217 & 0.00578625 & 0.00696899 \\
\hline
\end{tabular}

the left and for a positive value of $l$ it shifts towards the right. Deformation of the shape of the shadow is prominent in all cases.

Using the parameters, which are introduced by Hioki and Maeda in [9], we analyze the deviation from the circular form 

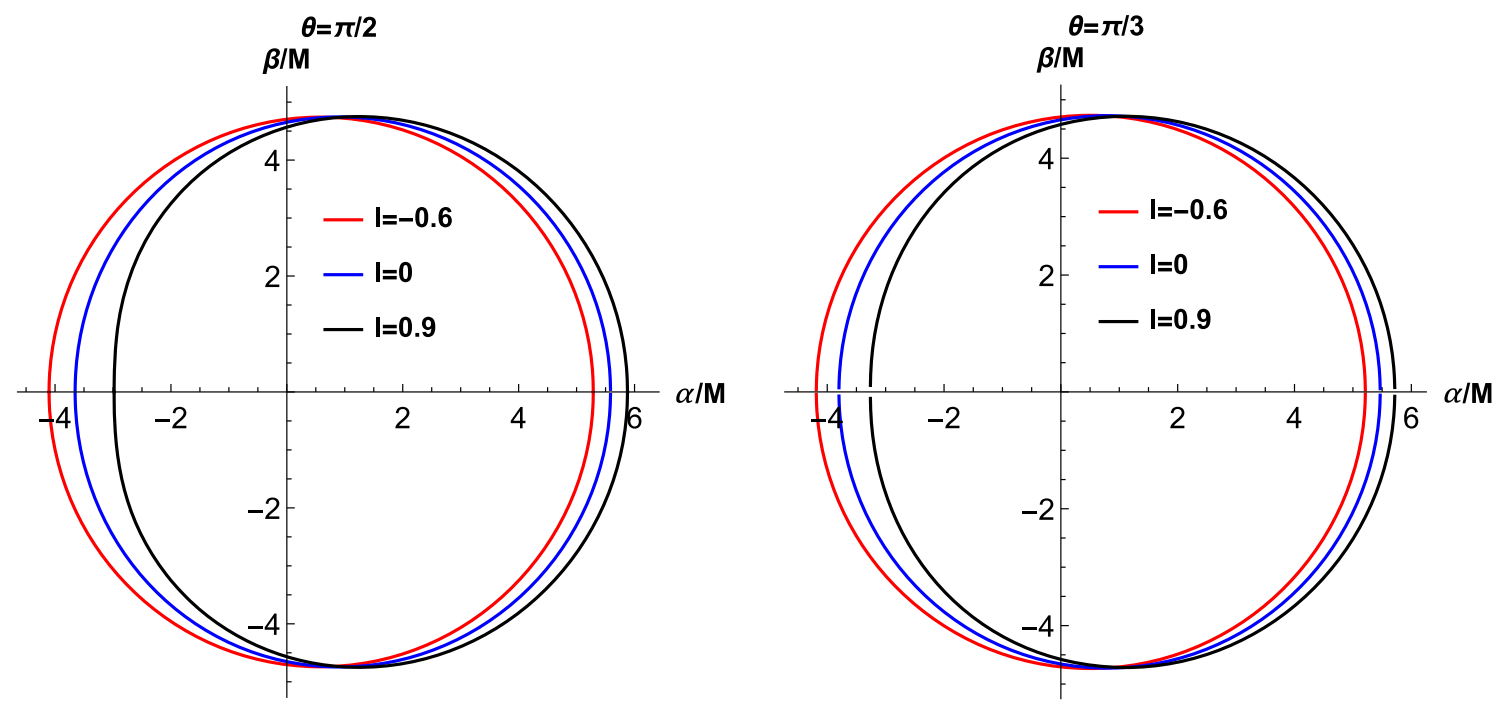

Fig. 7 The figure of the shadows for different values of $l$ when $\theta=\frac{\pi}{2}$ and $\theta=\frac{\pi}{3}$ with $a=0.4, b=0.5$, and $k=0.2$
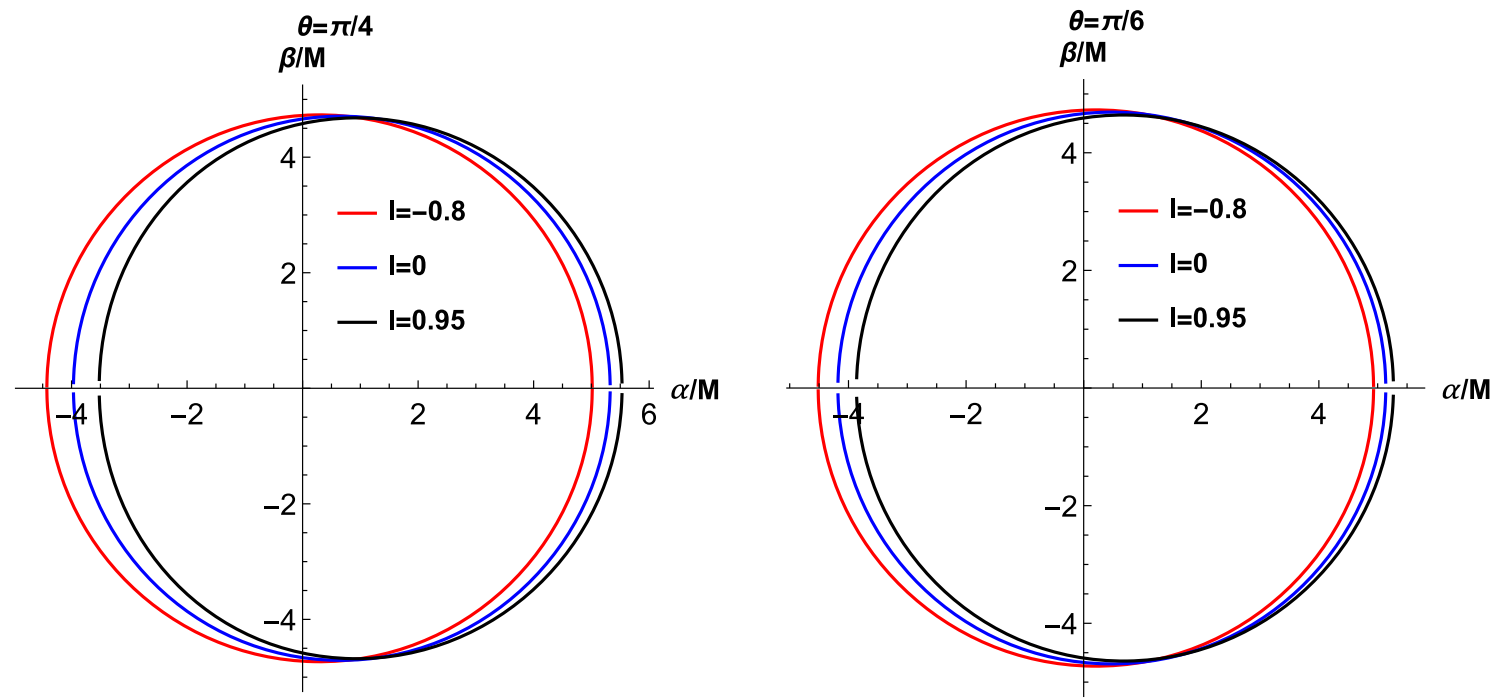

Fig. 8 The figure of the shadows for different values of $l$ when $\theta=\frac{\pi}{4}$ and $\theta=\frac{\pi}{6}$ with $a=0.4, b=0.5$, and $k=0.2$

of the shadow $\left(\delta_{S}\right)$ and the size $\left(R_{S}\right)$ of the shadow image of the black hole.

For calculating these parameters, we consider five points in the reference circle (Fig. 9). The four points $\left(\alpha_{t}, \beta_{t}\right)$, $\left(\alpha_{b}, \beta_{b}\right),\left(\alpha_{r}, 0\right)$ and $\left(\alpha_{p}, 0\right)$ are, respectively, the top, bottom, rightmost, and the leftmost point of the shadow; and $\left(\bar{\alpha}_{p}, 0\right)$ is the leftmost point of the reference circle. From simple geometry, we have expressions for the radius $R_{S}$ and the deviation from circularity $\delta_{s}$ as follows

$R_{s}=\frac{\left(\alpha_{t}-\alpha_{r}\right)^{2}+\beta_{t}^{2}}{2\left(-\alpha_{t}+\alpha_{r}\right)}$,

and

$\delta_{s}=\frac{\left(-\bar{\alpha}_{p}+\alpha_{p}\right)}{R_{s}}$.

For all subsequent plots, we have taken $\theta=\pi / 2$.
Here, we have given in a tabular form the deviation of the image $\delta_{s}$ of the Kerr-Sen-like black hole for set of values of $k \equiv\{0, .01, .02\}$ corresponding to the set of values of $k \equiv\{-.2,0, .2\}$ with $a=b=0.2$ and $\theta=\frac{\pi}{2}$. Figures 10 , 11,12 , are representing the plots of $R_{S}$ versus $b, k$ and $l$, and Figs. $13,14,15$ are containing the plots of $\delta_{s}$ versus $b, k$ and $l$.

\section{The rate of energy emission}

It is known that black holes emit radiation and consequently, the mass of the black hole decreases and the process continues until it collapses down completely [64]. For various black holes, this emission rate has been studied in the articles [65-67]. In [50], we have studied the energy emission rate 


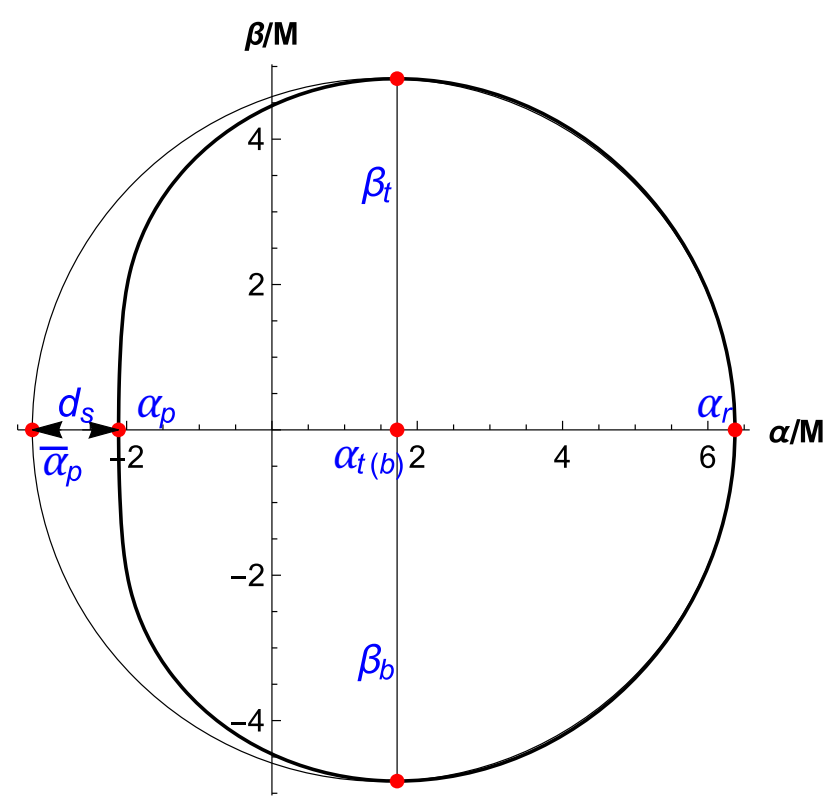

Fig. 9 A sketch of the black hole shadow and the reference circle. The distance between the extreme left point of the shadow, and the reference circle is represented by $d_{s}$

for Kerr-Sen-like black holes and studied the consequences of Lorentz violation effect $l$ on it. Here we would like to study the energy emission in presence of plasma following the motivation from the article [67]. The energy emission rate of radiation with the frequency $\omega$ is given by

$\frac{d^{2} E}{d \omega d t}=\frac{2 \pi^{3} R_{s}^{2}}{e^{\frac{\omega}{T}}-1} \omega^{3}$.

where $\omega$ is the frequency and $T$ is the Hawking temperature given by [50]

$T=\frac{\sqrt{(2 M-b)^{2}-4 a^{2}(1+l)}}{4 \pi M \sqrt{1+l}\left(2 M-b+\sqrt{(2 M-b)^{2}-4 a^{2}(1+l)}\right)}$.

In [65], it was conjectured that the black hole shadow corresponds to its high energy absorption cross-section for the observer located at infinity. In general, the absorption crosssection oscillates around a limiting constant value $\sigma_{\text {lim }}=$ $\pi R_{s}^{2}$ for a spherically symmetric black hole $[65,68,69]$. The reader may see the articles $[68,69]$ where it was shown that $\sigma_{\text {lim }}$ was proportional to the geometrical cross-section of its photon sphere. The fluctuation around the limiting value was also studied in [70]. In [66], it has been applied for studying energy emission. In [67], it was extended for the black hole in presence of plasma. Therefore, the energy emission rate is directly proportional to the surface area of the black hole, which may be considered to be approximately equal to the surface area of the shadow $S \approx \pi R_{s}^{2}$. Computing $R_{S}$ using the expression (26) and using (29) we can calculate the rate of emission of radiation, which enables us to plot energy emission versus frequency of radiation curve. In Fig. 16, we have studied how the energy emission rate will behave with the variation of the parameters $b, l$, and $k$. It is observed that the rate of emission is higher for smaller values of $b$. However, the reverse is the case when $k$ increases. It is evident from the spectrum that in the absence of plasma the minimum energy will be released from the black hole, which indeed agrees with the Kerr-Newman black hole [40]. From the plots, we see that for negative $l$ there is an enhancement of emission whereas, for increasing positive value of $l$, there is a reduction in the emission of radiation (Table 2).

\section{Angle of deflection of light in weak field in the presence of plasma}

The deflection of light or lensing in presence and in the absence of dispersive medium like plasma for different spacetime backgrounds has been studied in the earlier literature [71? -75]. The extension of it for this Kerr-Sen-like black hole is worth investigation. There is an LV parameter $l$. So from our study, the combined effect of the presence of plasma and Lorentz violation on the deflection of light can be estimated. To study it, weak field approximation will be useful. This approximation is given by the expression

$g_{\mu \nu}=\eta_{\mu \nu}+h_{\mu \nu}$,

where $\eta_{\mu v}$ is the Minkowski metric and $h_{\mu \nu}$ is the perturbation metric over $\eta_{\mu \nu}$. The Minkowski metric $\eta_{\mu \nu}=$ diag $(-1,1,1,1)$ and $h_{\mu \nu}$ has the the following properties under weak field approximation

$$
\begin{aligned}
& h_{\mu \nu} \ll 1, \quad h_{\mu \nu} \rightarrow 0 \quad \text { under } \quad x^{i} \rightarrow \infty, \\
& g^{\mu \nu}=\eta^{\mu \nu}-h^{\mu \nu}, \quad h^{\mu \nu}=h_{\mu \nu} .
\end{aligned}
$$

Considering the weak plasma strength, the angle of deflection of photon propagating along $z$ direction under weak field approximation is given by [77]

$\hat{\alpha}_{k}=\frac{1}{2} \int_{-\infty}^{\infty}\left(h_{33}+\frac{h_{00} \omega^{2}-K_{e} N}{\omega^{2}-\omega_{e}^{2}}\right)_{, k} d z$,

where $K_{e}=\frac{4 \pi e^{2}}{m}$. For large $\mathrm{r}$, the black hole metric can, approximately, be written down in the form

$$
\begin{gathered}
d s^{2}=d s_{0}^{2}+\left(\frac{2 M}{r}-\frac{2 M b}{r^{2}}\right) d t^{2} \\
+(1+l)\left(\frac{2 M}{r}-\frac{2 M b}{r^{2}}\right) d r^{2}
\end{gathered}
$$

where

$d s_{0}^{2}=-d t^{2}+(1+l) d r^{2}+r^{2}\left(d \theta^{2}+\sin ^{2} \theta d \phi^{2}\right)$. 

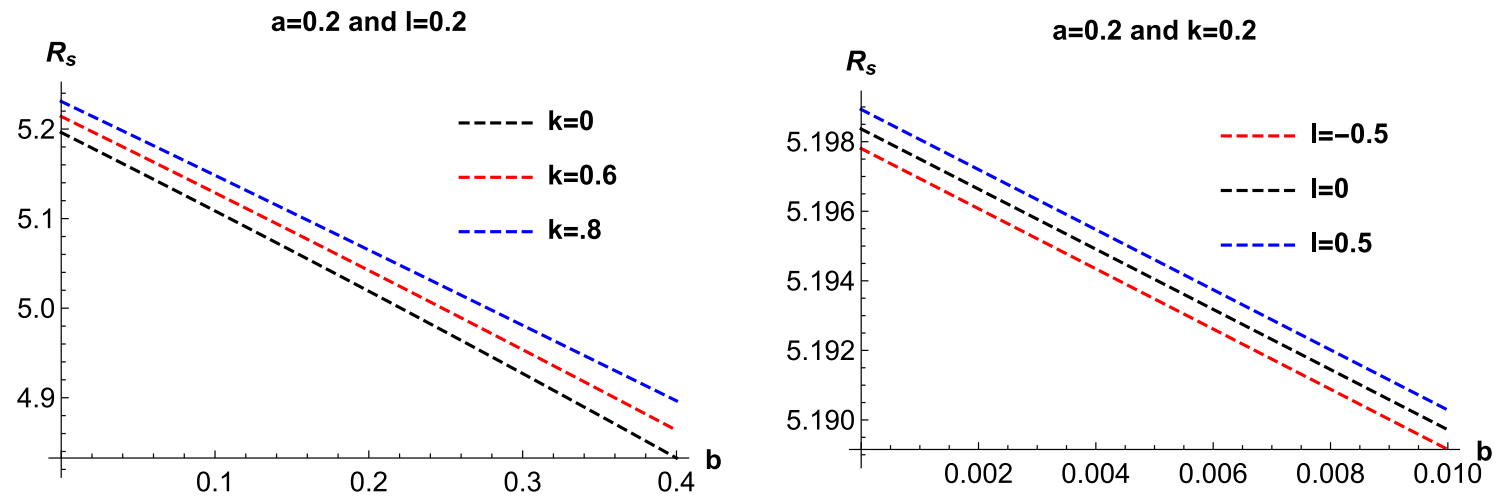

Fig. 10 The figure at the left side represents the variation of shadow radius $R_{s}$ with $b$ for different values of $k$ with $a=0.2$ and $l=0.2$. The figure at the right side represents the variation of $R_{s}$ with $b$ for different values of $l$ with $a=0.2$ and $k=0.2$
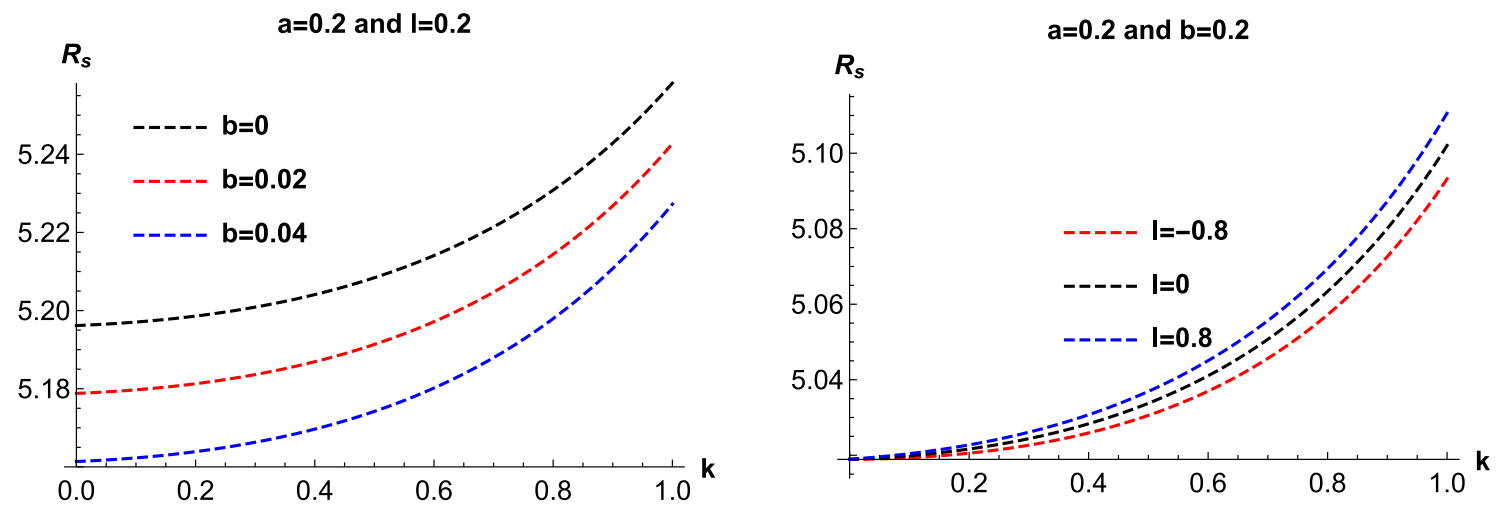

Fig. 11 The left one represents the variation of shadow radius $R_{s}$ with $k$ for different values of $b$ with $a=0.2$ and $l=0.2$. The right one represents the variation of $R_{s}$ with $k$ for different values of $l$ with $a=0.2$ and $b=0.2$
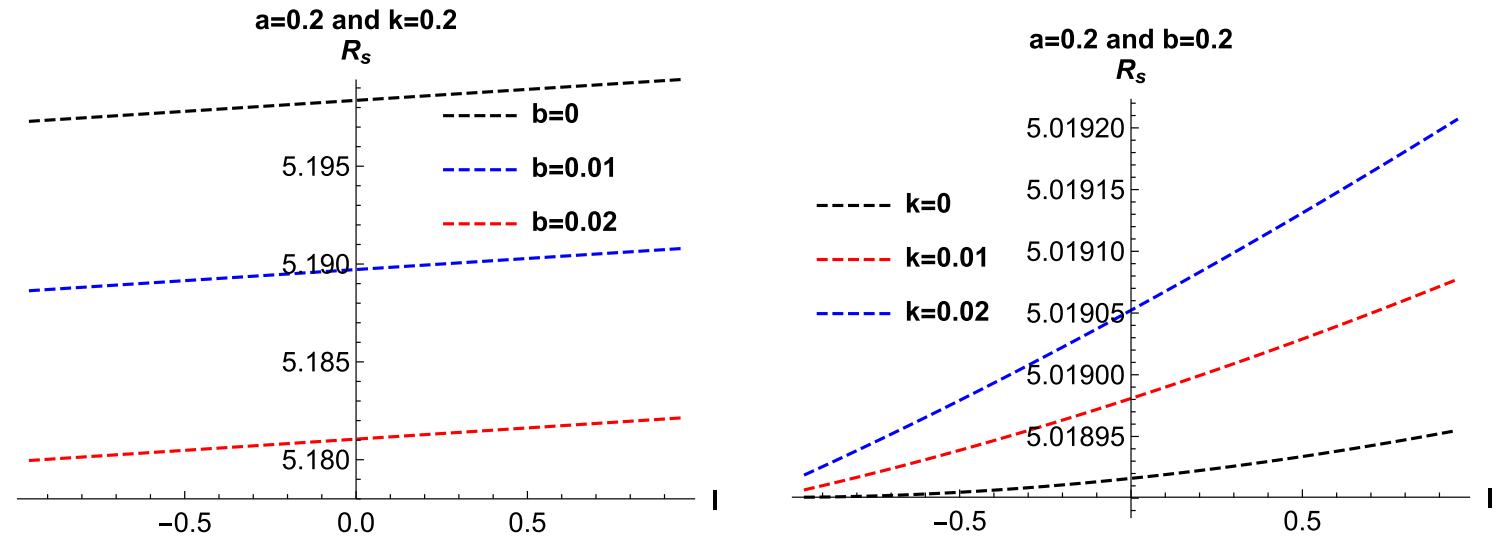

Fig. 12 The figure at the left side represent the variation of $R_{s}$ with $l$ for different values of $b$ with $a=0.2$ and $k=0.2$. The figure at the right side represents the variation of $R_{s}$ with $l$ for different values of $l$ with $a=0.2$ and $b=0.2$

The components $h_{\alpha \beta}$ in the Cartesian coordinates have the following expressions

$$
\begin{aligned}
h_{00} & =\left(\frac{R_{g}}{r}-\frac{2 M b}{r^{2}}\right), \\
h_{i k} & =(1+l)\left(\frac{R_{g}}{r}-\frac{2 M b}{r^{2}}\right) n_{i} n_{k},
\end{aligned}
$$

$$
h_{33}=(1+l)\left(\frac{R_{g}}{r}-\frac{2 M b}{r^{2}}\right) \cos ^{2} \chi,
$$

where $R_{g}=2 M$ and $\chi$ is the polar angle between 3 -vector and $\mathrm{z}$-axis. Using the above expressions in (32), the light 

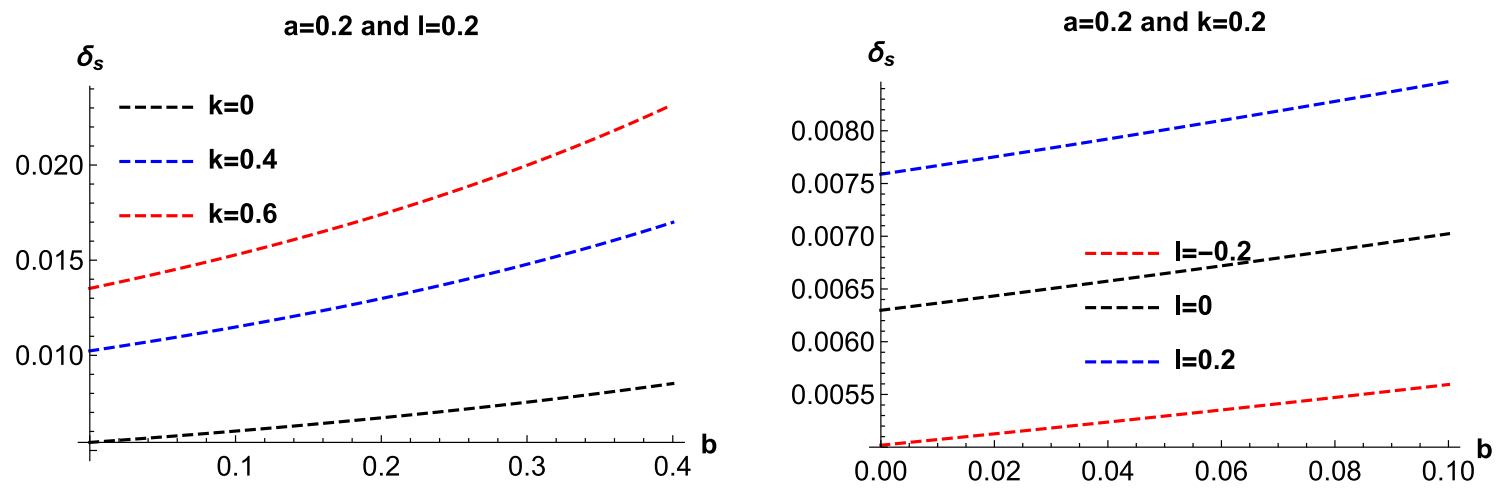

Fig. 13 The figure at the left side represent the variation of deviation from circularity $\delta_{s}$ with $b$ for different values of $k$ with $a=0.2$ and $l=0.2$. The figure at the right side represents the variation of $\delta_{s}$ with $b$ for different values of $l$ with $a=0.2$ and $k=0.2$
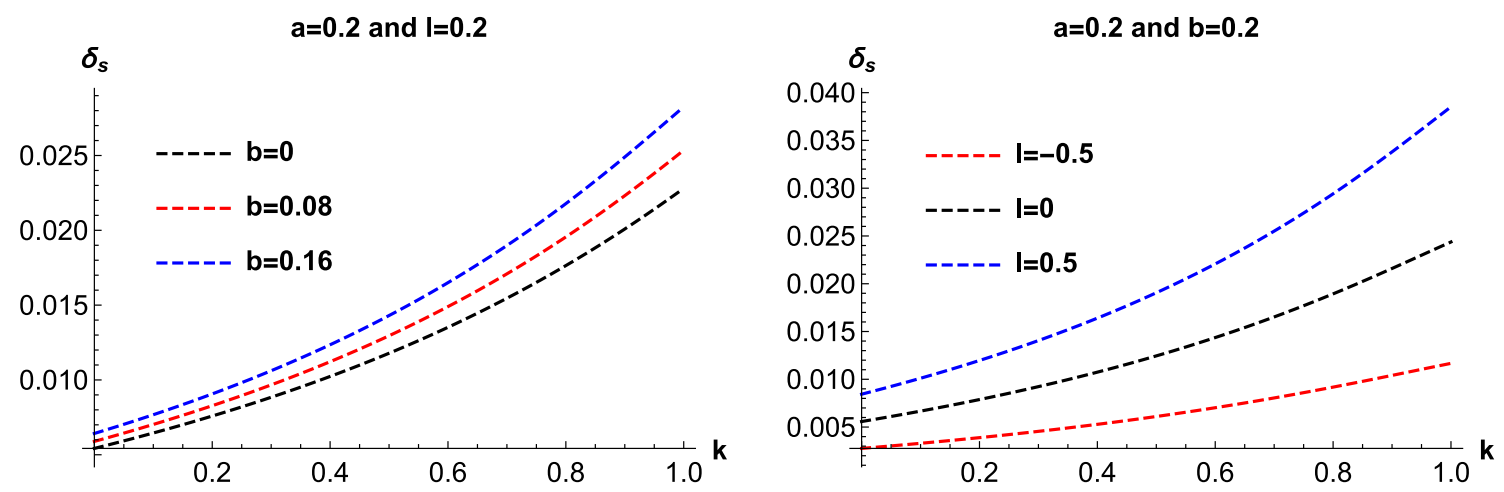

Fig. 14 The figure at the left side represents the variation of deviation from circularity $\delta_{s}$ with $k$ for different values of $b$ with $a=0.2$ and $l=0.2$. The figure at the right side represents the variation of $\delta_{s}$ with $k$ for different values of $l$ with $a=0.2$ and $b=0.2$
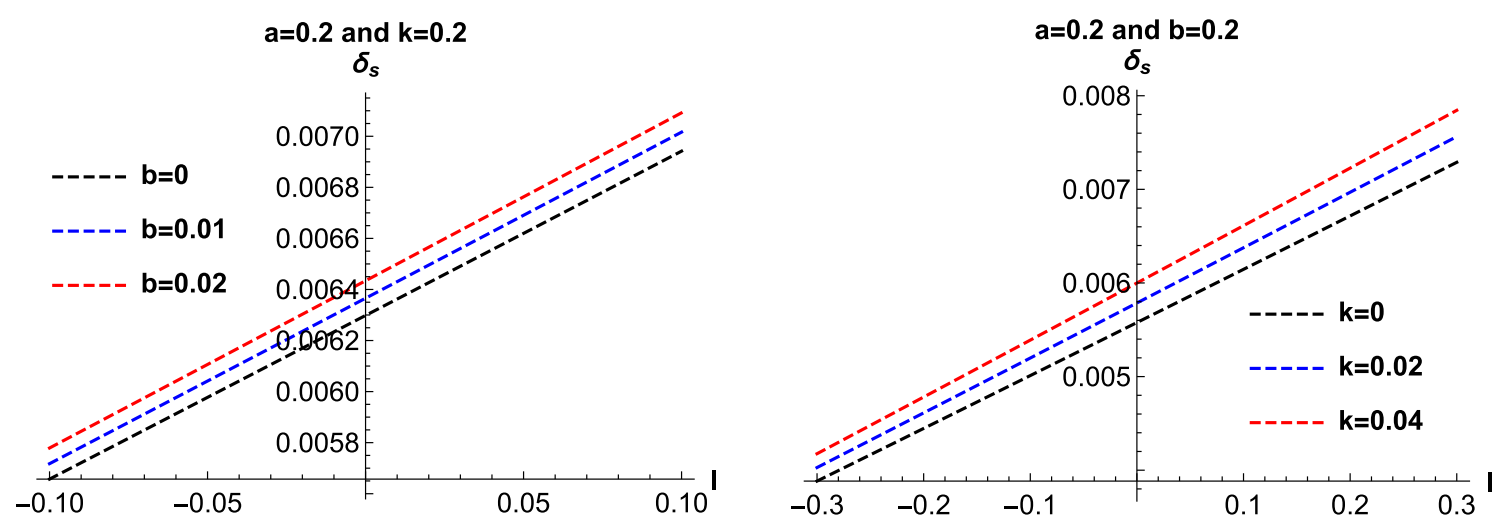

Fig. 15 The figure at the left side represents the variation of deviation from circularity $\delta_{s}$ with $l$ for different values of $b$ with $a=0.2$ and $k=0.2$. The figure at the right side represents the variation of $\delta_{s}$ with $l$ for different values of $l$ with $a=0.2$ and $b=0.2$

deflection angle for a black hole sitting in plasma medium $[71-73,78]$ is given by

$\hat{\alpha}_{p}=\frac{1}{2} \int_{-\infty}^{\infty} \frac{p}{r}\left(\frac{d h_{33}}{d r}+\frac{\omega^{2}}{\omega^{2}-\omega_{e}^{2}} \frac{d h_{00}}{d r}-\frac{K_{e}}{\omega^{2}-\omega_{e}^{2}} \frac{d N}{d r}\right) d z$, where $p^{2}=x_{1}^{2}+x_{2}^{2}$ refers to the impact parameter and $x_{1}$ and $x_{2}$ are the coordinates on the plane orthogonal to the $z$ axis. The expression of $r$ is $r=\sqrt{p^{2}+z^{2}}$. We have the relation for the frequency of photon

$\omega^{2}=\frac{\omega_{\infty}^{2}}{\left(1-\frac{R_{g}}{r}+\frac{2 M b}{r^{2}}\right)}$. 


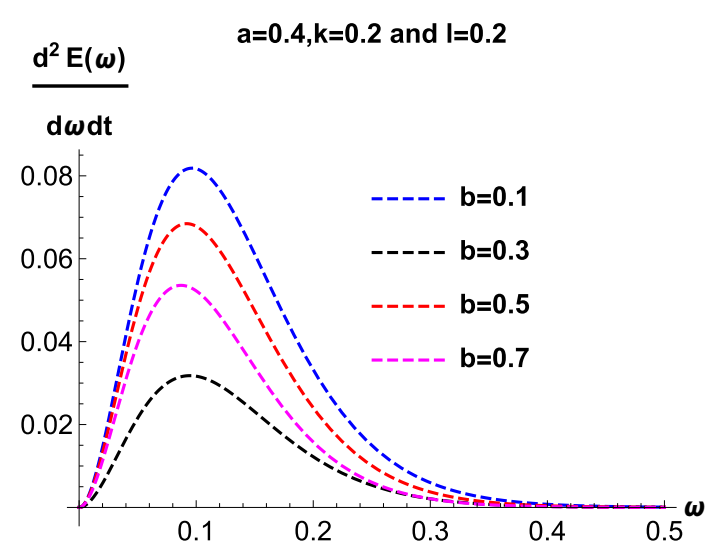

(a) Variation of the rate of emission with $\omega$ for different values of $b$ with $a=0.4, k=0.2$, and $l=0.2$.

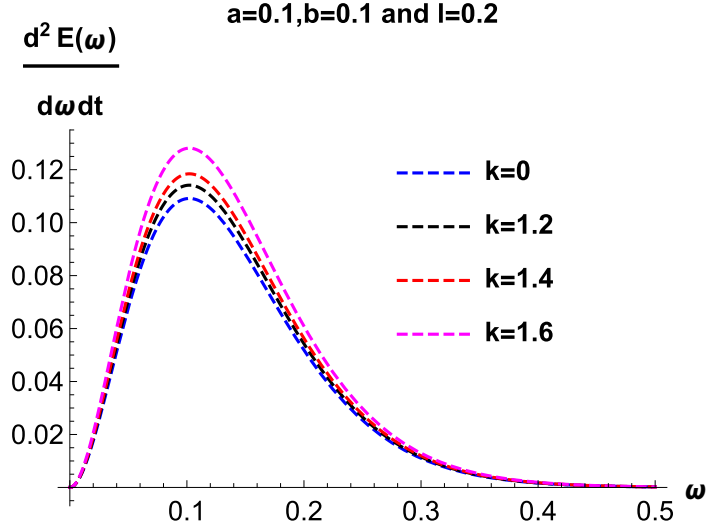

(b) Variation of the rate of emission with $\omega$ for different values of $k$ with $a=0.1, b=0.1$, and $l=0.2$.

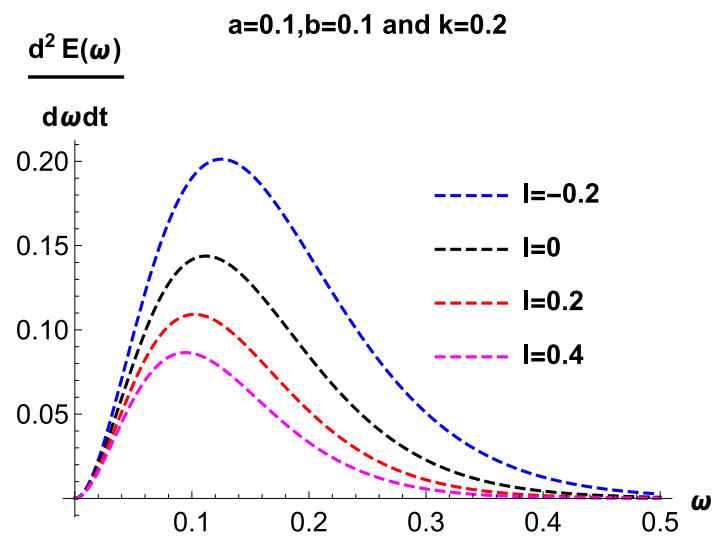

(c) Variation of the rate of emission with $\omega$ for

different values of $l$ with $a=0.1, b=0.1$, and $k=0.2$.

Fig. 16 Variation of the rate of emission with $\omega$ for different situations

Here $\omega_{\infty}$ is the asymptotic value of photon frequency. After expanding in series on the powers of $\frac{1}{r}$, we can have the approximation

$$
\left(1-\frac{\omega_{e}^{2}}{\omega^{2}}\right)^{-1} \simeq 1+\frac{4 \pi e^{2} N_{0} r_{0}}{m \omega_{\infty}^{2} r}-\frac{4 \pi e^{2} N_{0} r_{0} R_{g}}{m \omega_{\infty}^{2} r^{2}} .
$$

Using this approximation, one can find the deflection angle $\hat{\alpha}_{p}$ of the light around a black hole in presence of plasma in a straightforward manner

$$
\begin{aligned}
\hat{\alpha}_{p}= & (1+l) \frac{2 R_{g}}{p}+\frac{2 R_{g}}{p}\left(1+\frac{\pi^{2} e^{2} N_{0} r_{0}}{m \omega_{\infty}^{2} p}-\frac{4 \pi e^{2} N_{0} r_{0} R_{g}}{m \omega_{\infty}^{2} p^{2}}\right) \\
& -\frac{2 M b}{4 p^{2}}\left(3 \pi+\frac{4 \pi e^{2} N_{0} r_{0}}{m \omega_{\infty}^{2} p}\left(8-\frac{3 \pi R_{g}}{p}\right)\right) .
\end{aligned}
$$

We get $\hat{\alpha}_{p}=(1+l) \frac{2 R_{q}}{p}$ in the absence of charge and plasma for the Schwarzschild black hole. The dependence of the angle of deflection $\hat{\alpha}_{p}$ on the impact parameter $p$ for various charge and plasma parameters are demonstrated in the figures below where $F=\frac{4 \pi e^{2} N_{0} r_{0}}{m \omega_{\infty}^{2}}$. In the left panel, it is found that as the value of $b$ increases the angle of deflection decreases and it is seen that $\hat{\alpha}_{p}$ is maximum when $b=0$. We also observe that the deflection angle $\hat{\alpha}_{p}$ increases with the increase in the plasma parameter (right panel). We also notice that the deviation of photons is smaller when the plasma factor is removed from the black hole background. It is also observed that the deflection angle increases with an increase in the value of the Lorentz violating parameter $l$ (Fig. 17).

\section{Constraining the parameter from the observed data for M87*}

We compare the shadows produced, from numerical calculations, by the Kerr-Sen-like black holes with the observed one for the M87* black hole. For comparison we consider the experimentally obtained astronomical data for the circularity deviation $\Delta \leq 0.10$ and angular diameter $\theta_{d}=42 \pm 3 \mu$ as [2]. The boundary of the shadow is described by the polar coordinate $(R(\phi), \phi)$ with the origin at the center of the shadow $\left(\alpha_{C}, \beta_{C}\right)$ where $\alpha_{C}=\frac{\left|\alpha_{\max }+\alpha_{\min }\right|}{2}$ and $\beta_{C}=0$. 

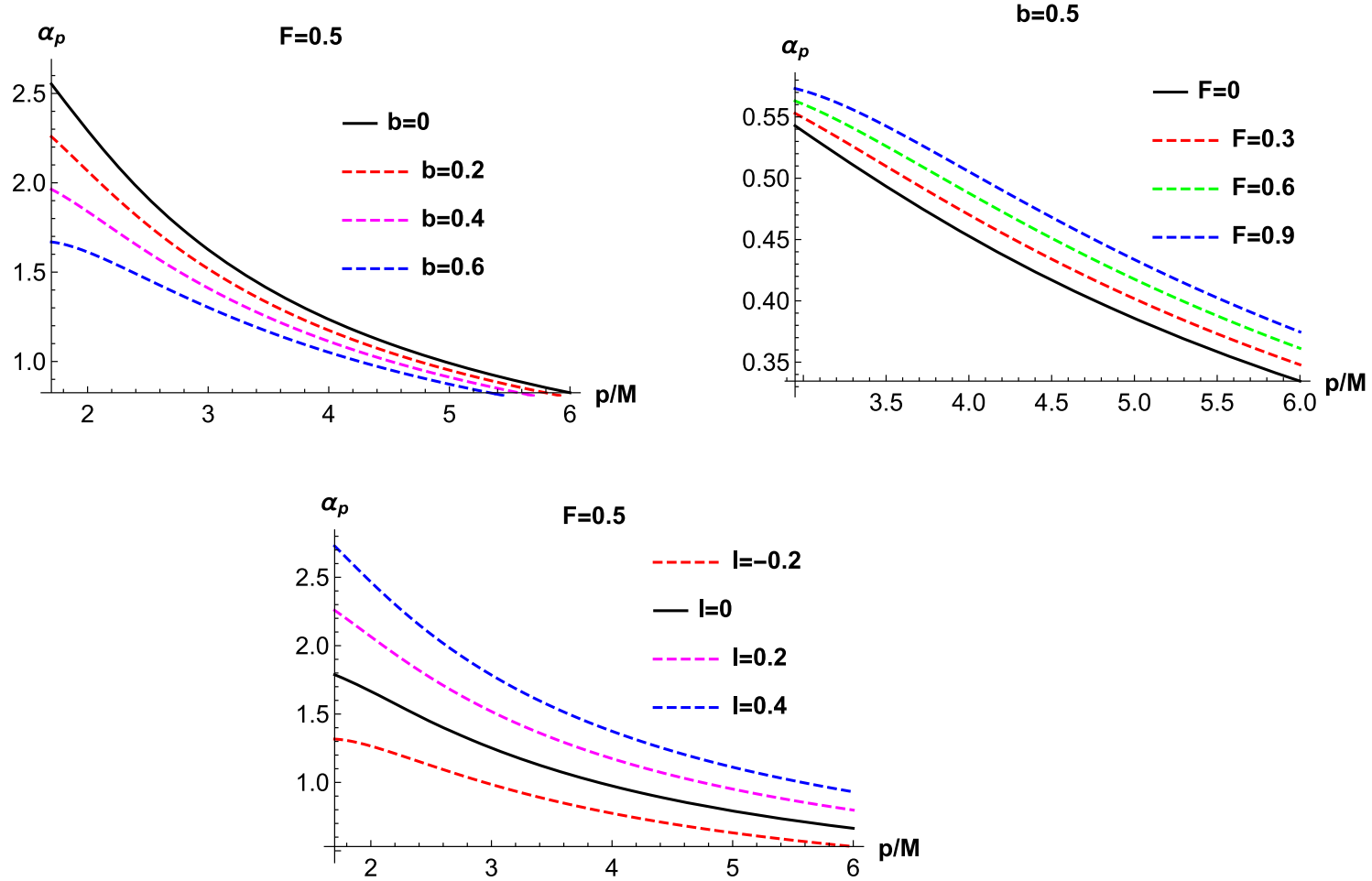

Fig. 17 The plot at upper left position represents the variation of angle of deflection $\hat{\alpha}_{p}$ with $p / M$ for different values of $b$ with $F=\frac{4 \pi e^{2} N_{0} r_{0}}{m \omega_{\infty}^{2}}=0.5$ and $l=0.2$. The plot at upper right position describes the variation of $\hat{\alpha}_{p}$ with $p / M$ for different values

If a point $(\alpha, \beta)$ over the boundary of the image subtends an angle $\phi$ on the $\alpha$ axis at the geometric center, $\left(\alpha_{C}, 0\right)$ and $R(\phi)$ be the distance between the point $(\alpha, \beta)$ and $\left(\alpha_{C}, 0\right)$, then the average radius $R_{\text {avg }}$ of the image is given by [80]

$R_{\mathrm{avg}}^{2} \equiv \frac{1}{2 \pi} \int_{0}^{2 \pi} d \phi R^{2}(\phi)$,

where $R(\phi) \equiv \sqrt{\left(\alpha(\phi)-\alpha_{C}\right)^{2}+\beta(\phi)^{2}}$, and $\phi=\tan ^{-1}$ $\frac{\beta(\phi)}{\alpha(\phi)-\alpha_{C}}$.

With the above inputs, the deviation from circularity $\Delta C$ is defined by [79],

$\Delta C \equiv 2 \sqrt{\frac{1}{2 \pi} \int_{0}^{2 \pi} d \phi\left(R(\phi)-R_{\mathrm{avg}}\right)^{2}}$.

We also consider the shadow angular diameter which is define by

$\theta_{d}=\frac{2}{d} \sqrt{\frac{A}{\pi}}$

where $A=2 \int_{r_{-}}^{r_{+}} \beta d \alpha$ is the shadow area and $d=16.8 M p c$ is the distance of $M 87^{*}$ from the earth. These relations will enable us to accomplish a comparison between the theoretical predictions for Kerr-Sen-like black-hole shadows and of $F=\frac{4 \pi e^{2} N_{0} r_{0}}{m \omega_{\infty}^{2}}$ with $b=0.5$ and $l=-0.4$. The lower one represents the variation of $\hat{\alpha}_{p}$ with $p / M$ for different values of $l$ with $F=\frac{4 \pi e^{2} N_{0} r_{0}}{m \omega_{\infty}^{2}}=0.5$ and $b=0.2$

the experimental findings of the Event Horizon Telescope collaboration. In Table 2 the values of deviation $\Delta C$ of KerrSen-like black hole with $k=l=0.1$ for the inclination angle $\theta=90^{\circ}$ and $\theta=170^{\circ}$ are computed. Figures below represent the deviation from circularity $\Delta C$ as it is obtained for Kerr-Sen-like black holes for the angles of inclinations $\theta=90^{\circ}$ and $17^{\circ}$ respectively.

Figures below represent the angular diameter $\theta_{d}$ as it is obtained for Kerr-Sen-like black holes for the angles of inclinations $\theta=90^{\circ}$ and $17^{\circ}$ respectively.

For this type of estimation, a prerequisite independent input is the knowledge of the black hole mass, which is the intrinsic yardstick for this system. In the case of M87*, the mass has been estimated to be $M=(6.6 \pm 0.4) \times 10^{9} M_{\odot}$ [81]. Considering $M=6.5 \times 10^{9} M_{\odot}$ for the $\mathrm{M} 87^{*}$ black hole, we use the observed value of deviation from circularity $\Delta C \leq 0.10$ as deduced by the EHT collaboration [2] in order to constrain the Kerr-Sen-like black hole parameters. We have made an attempt to put constraints on $(a, k),(a, b)$ and $(a, l)$ by fixing the values of $(b, l),(k, l)$ and $(b, k)$ respectively. From Figs. 18 and 19, it is clear that over a finite parameter space, black hole shadows satisfy the bound $\Delta C \leq 0.10$. Figures 20 and 21 reveal that the condition $\Delta C \leq 0.10$ is satisfied by the Kerr-Sen-like black hole in presence of plasma 

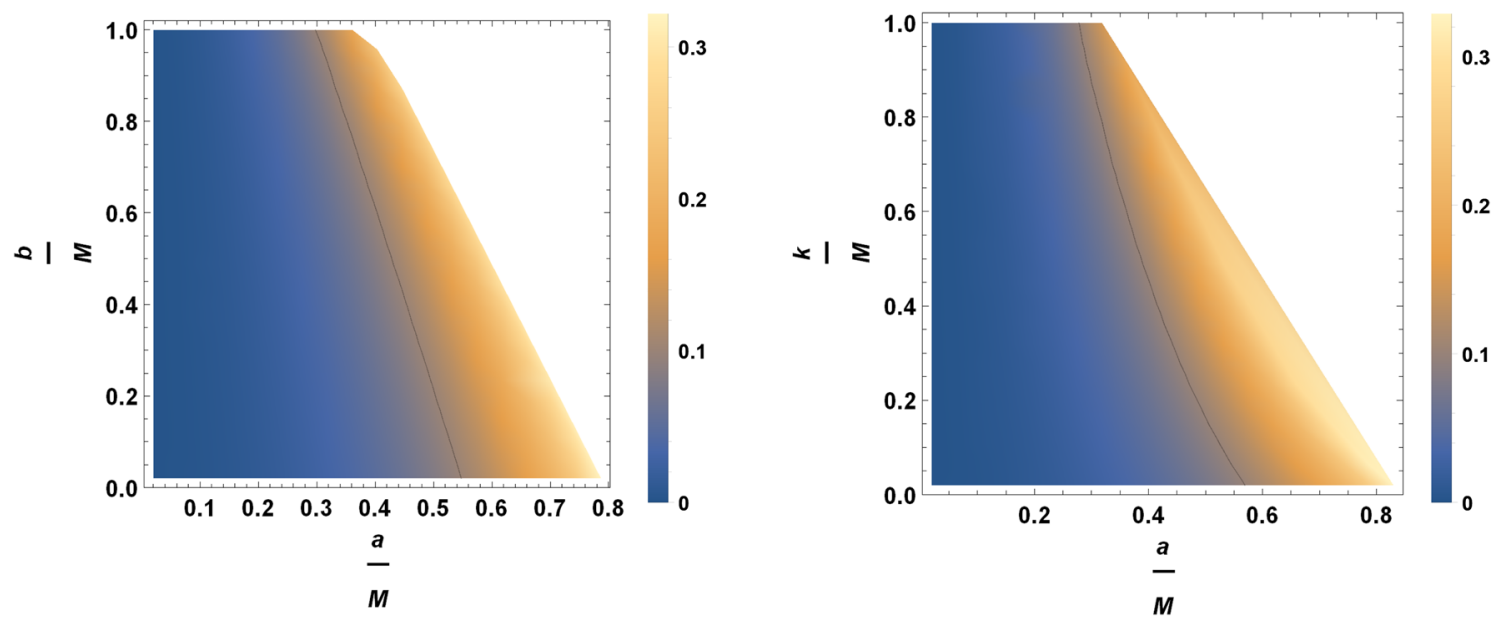

Fig. 18 The observable for deviation from circularity $\Delta C$ as a function of black hole parameters. The left one is for $l=0.1$ and $k=0.1$. The right one is for $l=0.1$ and $b=0.1$. The angle of inclination is $\theta=90^{\circ}$. The black lines in the figures correspond to $\Delta C=0.1$
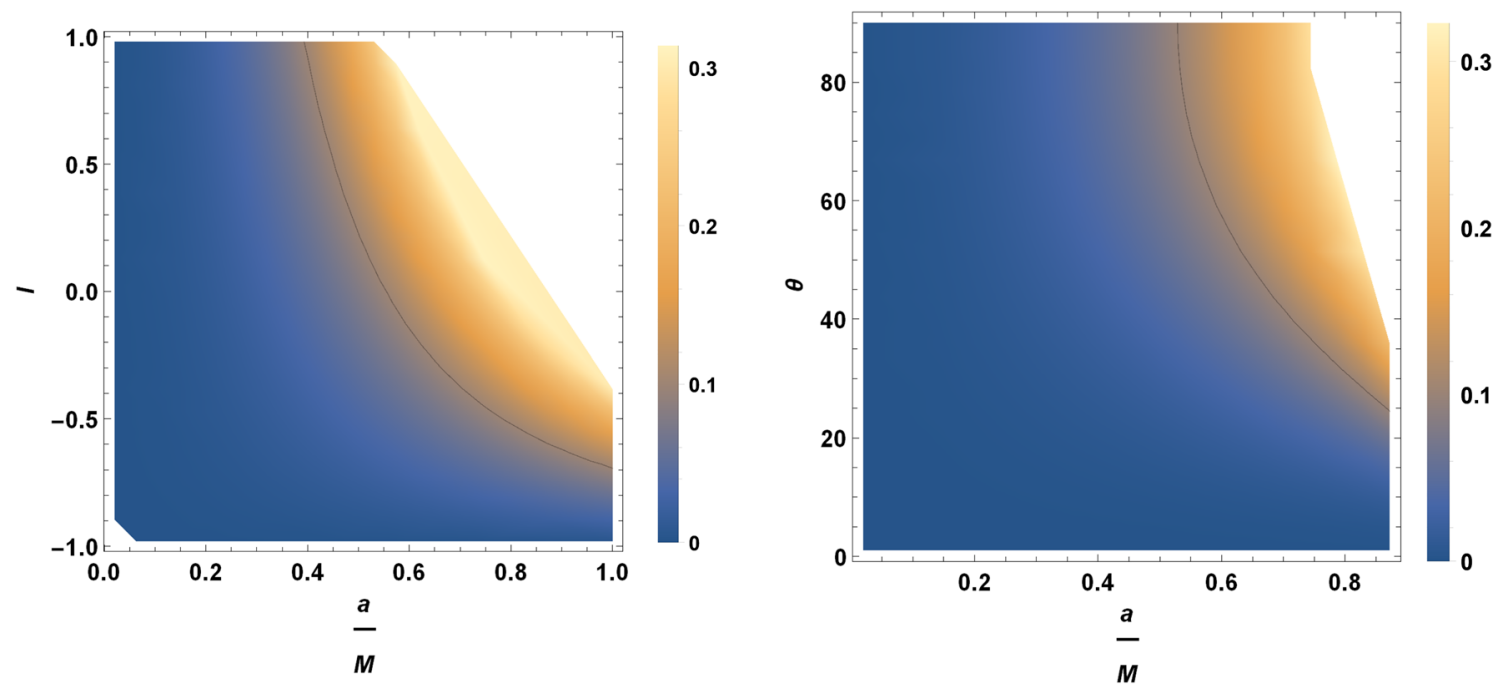

Fig. 19 The observable for deviation from circularity $\Delta C$ as a function of black hole parameters. The left one is for $b=0.1$ and $k=0.1$. The right one is for $l=0.1, b=0.1$, and $k=0.1$. Here the angle of inclination is $\theta=90^{\circ}$. The black lines in the figures correspond to $\Delta C=0.1$

for the entire parameter space. From Figs. 22, 23 and 24 it is clear that, for finite parameter space, angular diameters are within $1 \sigma$ region i.e $\theta_{d}=39 \mu$ as. From figures above we can also conclude that for fixed values of black hole parameters, the apparent shadow circularity deviation and angular diameter decrease as the observer moves away from the equatorial plane toward the polar axis.

We may put constrain on the value of $l$ as well. By modelling M87* black hole as Kerr black hole, the author has found a lower limit a $a$ for the M87* black hole [82]. If we bring this result under consideration in the present situation the interval of interest for $a$ becomes [0.50M, 0.99M] with $b=k=0$. Now if we combine the constraints $\Delta C \leq 0.10$ and $\theta_{d}=42 \pm 3 \mu a s$ and the knowledge that $a \in[0.50 M, 0.99 M]$, we observe that $l \in(-1,0.621031]$. Here we find upper bound of $l$ to be 0.621031 , however $l$ cannot be equal to -1 . So $l>-1$. So to consider both the positive and negative value of $l$ in every plot make sense and well justified

\section{Conclusion}

We have considered the Kerr-Sen-like black hole, which is a solution of Einstein-bumblebee gravity. It contains an LV parameter $l$. We study the propagation of light in a nonmagnetized pressure-less plasma on this Kerr-Sen-like spacetime. We have considered the plasma as a medium with dispersive properties given by a frequency-dependent index of refraction. The gravitational field is determined by the mass, the spin, and the bumblebee parameter. The Gravitational field due to plasma is neglected here. We have studied how the 

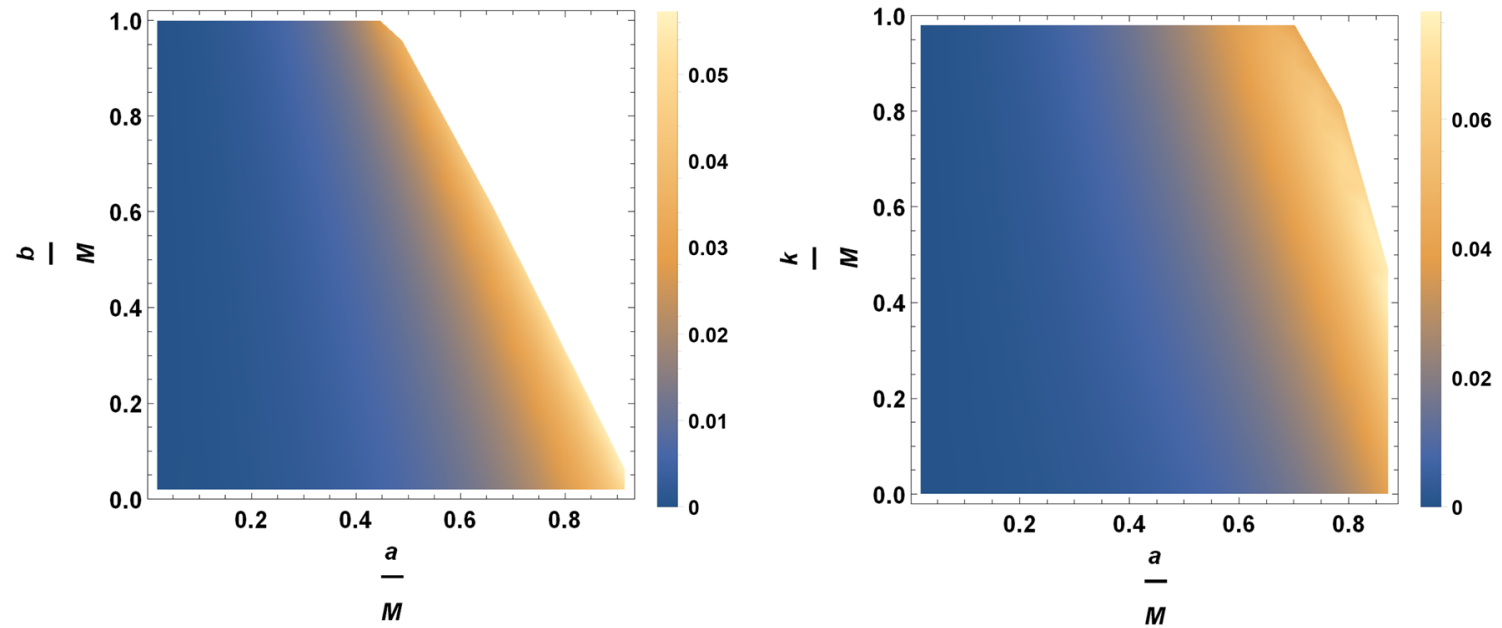

Fig. 20 The observable for deviation from circularity $\Delta C$ as a function of black hole parameters. The left one is for $l=0.1$ and $k=0.1$. The right one is for $l=0.1$ and $b=0.1$. Here the angle of inclination is $\theta=17^{\circ}$

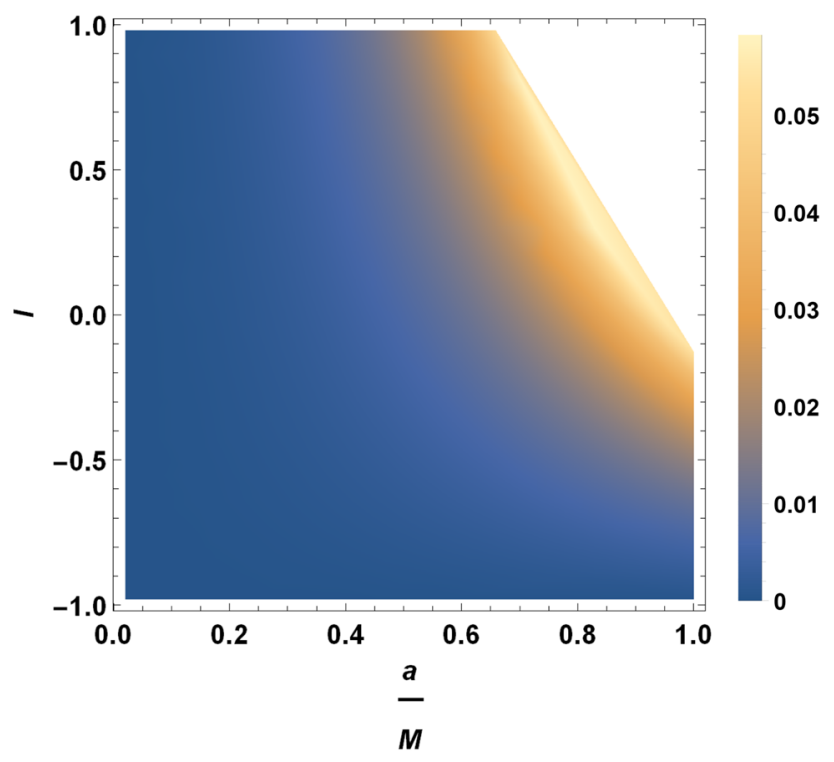

Fig. 21 The observable for deviation from circularity $\Delta C$ as a function of black hole parameters. This is for $b=0.1$ and $k=0.1$ with the angle of inclination $\theta=17^{\circ}$

nature of shadows gets affected by the LV effect associated with the bumblebee field in the pressure-less nonmagnetic plasma. We have also studied the energy emission scenario and the weak field lensing in the Kerr-Sen-like spacetime due to Einstein-bumblebee gravity background when it is veiled in a plasma medium. The results for Kerr, Kerr-like, and the Schwarzschild black holes can, as well, be obtained from this result with a suitable limit. We have investigated in detail the impact of the charge $\left(Q^{2} \propto b\right)$, plasma parameter $k$, and the Lorentz violating bumblebee parameter $l$ on the structure of shadow, on the lensing effect of light, and on the energy emission due to black hole radiation. It has been found that the new parameters quantitatively influence the structure of the event horizon by altering the radius significantly. It is observed that the size of the shadow viewed by a distant observer reduces with an increase in the charge $Q$ and the size of the shadow appears to be larger with the increase in the plasma parameter $k$ in the presence of a bumblebee field.

Therefore, the bumblebee field maintains its action of deforming the shadow in presence of plasma. Since the energy liberated from the black hole depends on the area of the shadow, the rate of energy emission from the black hole is higher when the black hole is surrounded by a plasma. As far as the angle of deflection is concerned, the photons are observed to experience an increase in the deviation as the plasma factor $k$ increases. While on the other hand, the angle of deflection reduces sufficiently when the amount of charge parameter increases. It is also observed that the deflection angle increases with an increase in the value of the LV parameter $l$. Sufficient negative $l$ may cease the lensing effect too!

The theory of General Relativity predicts that the Kerr metric is capable of describing astrophysical black holes. However, such black holes may not exist in a perfect vacuum due to the presence of surrounding matter, like plasma, dark matter, etc. The accretion disks may also alter the black hole, which may not fit with the Kerr metric. The bumblebee modified Kerr spacetime enables the incorporation of potential deviations from the Kerr metric through the additional deviation parameters. The advantage of this type of modified gravity is that it is a generic one and it has the ability to cover the prediction of the Kerr metric since the introduced parameters can be chosen in such a way that it makes the null deviation of the shadow from Kerr spacetime. There is the theoretical motivation for modified theories of gravity indeed. 

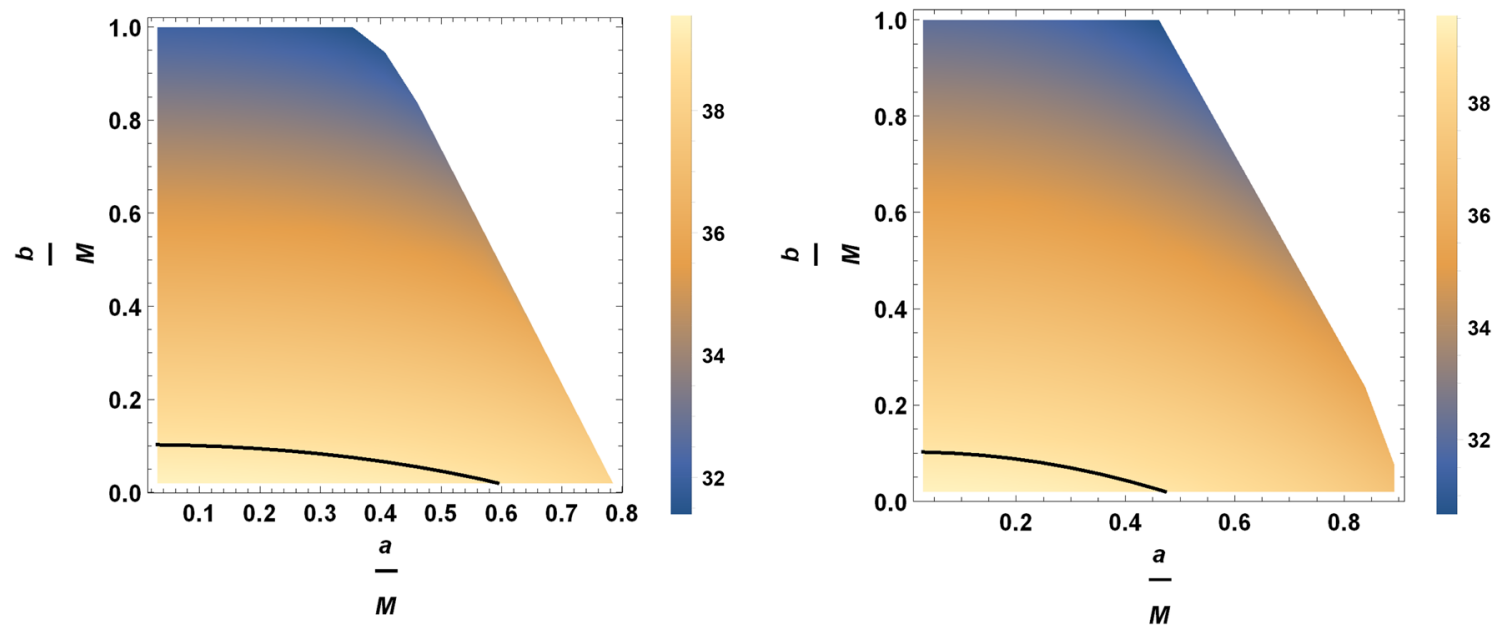

Fig. 22 The observable for angular diameter $\theta_{d}$ as a function of black hole parameters. The left one is for $l=0.1, k=0.1$, and angle of inclination $\theta=90^{\circ}$. The right one is for $l=0.1, k=0.1$, and $\theta=17^{\circ}$. Black solid lines correspond to $\theta_{d}=39 \mu a s$
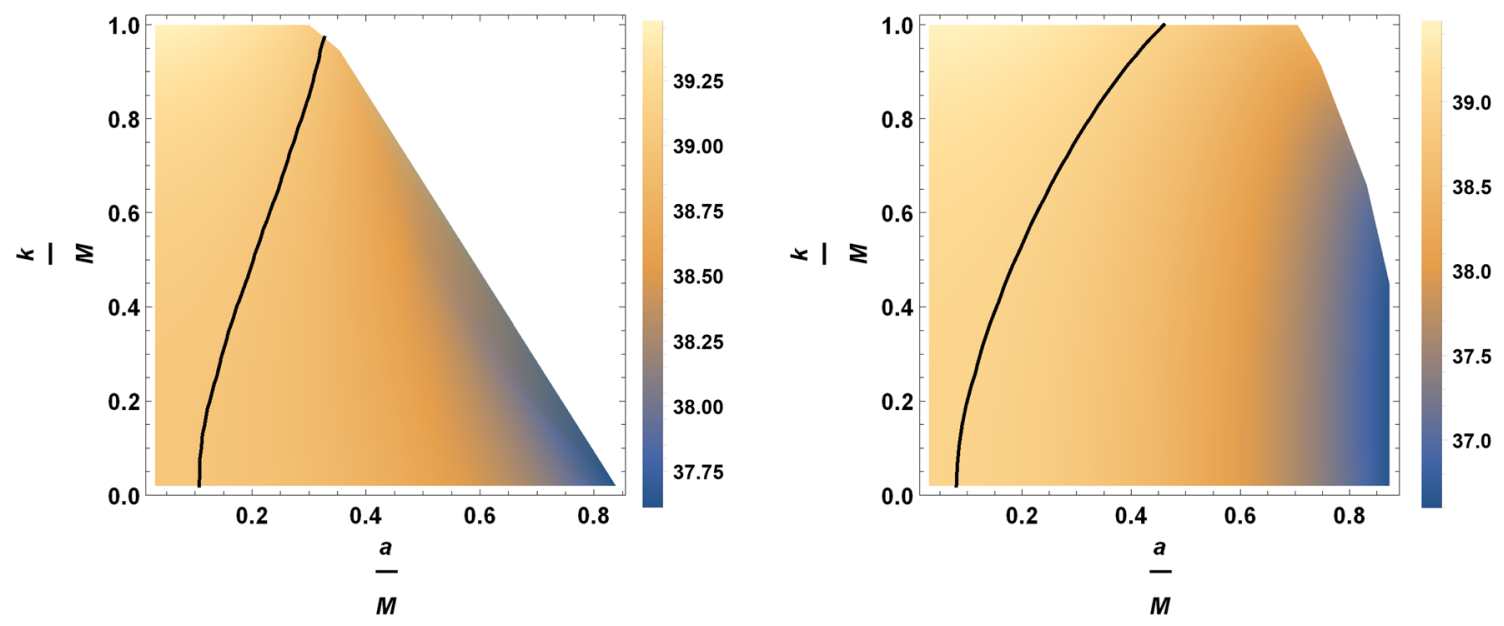

Fig. 23 The observable for angular diameter $\theta_{d}$ as a function of black hole parameters. The left one is for $l=0.1, b=0.1$, and angle of inclination $\theta=90^{\circ}$. The right one is for $l=0.1, b=0.1$, and $\theta=17^{\circ}$. Black solid lines correspond to $\theta_{d}=39 \mu$ as
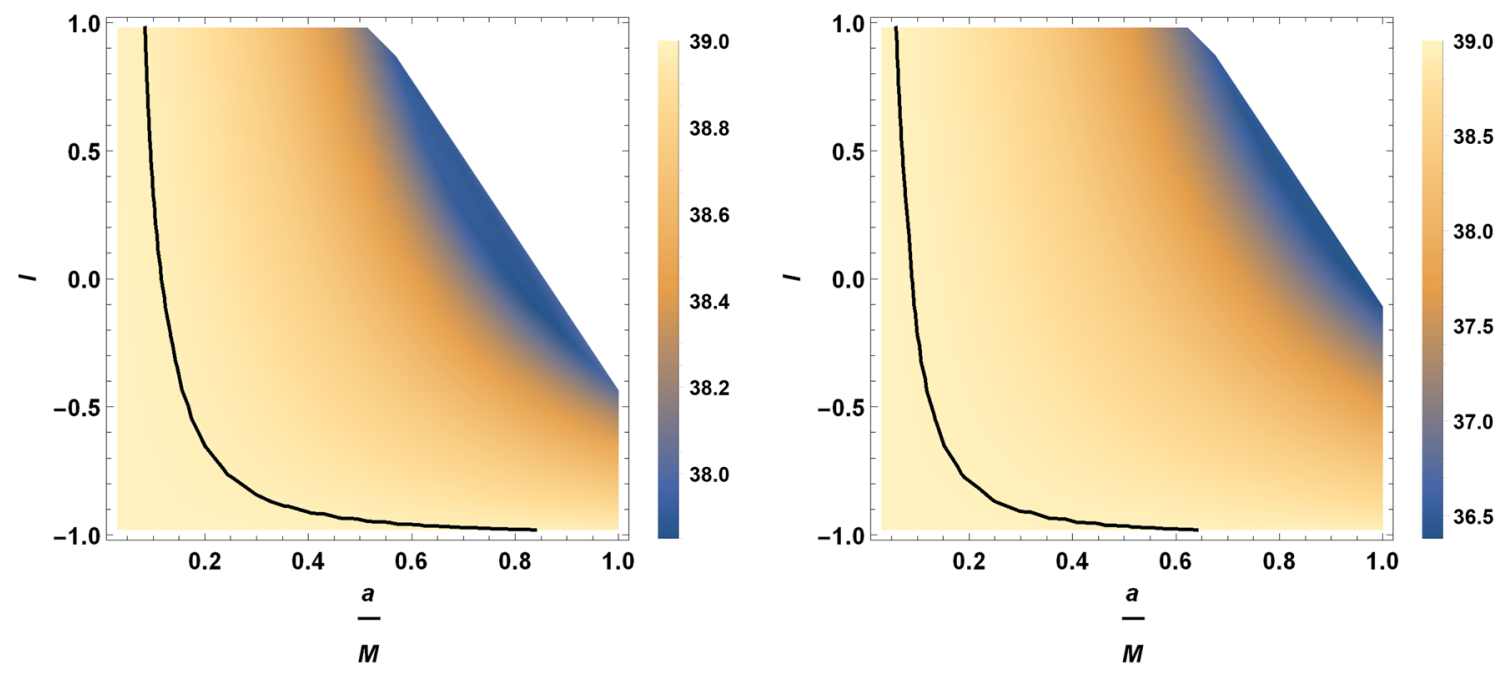

Fig. 24 The observable for angular diameter $\theta_{d}$ as a function of black hole parameters. The left one is for $k=0.1, b=0.1$, and angle of inclination $\theta=90^{\circ}$. The right one is for $k=0.1, b=0.1$, and $\theta=17^{\circ}$. Black solid lines correspond to $\theta_{d}=39 \mu$ as 
Table 2 Deviation $\Delta C$ of Kerr-Sen-like black hole with $k=l=0.1$. The upper one is for the inclination angle $\theta=90^{\circ}$ and the lower one is for the inclination angle $\theta=17^{\circ}$

\begin{tabular}{|c|c|c|}
\hline $\mathrm{a}$ & $\mathrm{b}$ & $\Delta C$ \\
\hline 0.020 & 0.020 & 0.000108539 \\
\hline 0.020 & 0.265 & 0.000135858 \\
\hline 0.020 & 0.510 & 0.000175677 \\
\hline 0.020 & 0.755 & 0.000237773 \\
\hline 0.020 & 1.000 & 0.000343416 \\
\hline 0.265 & 0.020 & 0.019818100 \\
\hline 0.265 & 0.265 & 0.025141500 \\
\hline 0.265 & 0.510 & 0.033223300 \\
\hline 0.265 & 0.755 & 0.046671500 \\
\hline 0.265 & 1.000 & 0.072912500 \\
\hline 0.510 & 0.020 & 0.085970200 \\
\hline 0.510 & 0.265 & 0.112077000 \\
\hline 0.510 & 0.510 & 0.165146000 \\
\hline $\mathrm{a}$ & $\mathrm{b}$ & $\Delta C$ \\
\hline 0.020 & 0.020 & 0.0000106680 \\
\hline 0.020 & 0.265 & 0.0000132214 \\
\hline 0.020 & 0.510 & 0.0000154633 \\
\hline 0.020 & 0.755 & 0.0000203415 \\
\hline 0.020 & 1.000 & 0.0000294412 \\
\hline 0.265 & 0.020 & 0.0017091800 \\
\hline 0.265 & 0.265 & 0.0021694100 \\
\hline 0.265 & 0.510 & 0.0028655300 \\
\hline 0.265 & 0.755 & 0.0040104600 \\
\hline 0.265 & 1.000 & 0.0061582600 \\
\hline 0.510 & 0.020 & 0.0073562800 \\
\hline 0.510 & 0.265 & 0.0097972700 \\
\hline 0.510 & 0.510 & 0.0140271000 \\
\hline
\end{tabular}

The EHT collaboration has captured the image of supermassive black hole M87* exhibiting a deviation from circularity $\Delta C \leq 0.10$. The observation did not say anything about modified theories of gravity or suggest any alternatives to the Kerr black hole. Motivated by this, we considered KerrSen-like modified black holes, which have additional deviation parameters $l$ and $b$ to the Kerr black hole. The presence of Plasma adds an additional parameter. We use the deviation from circularity $\Delta C \leq 0.10$ as deduced by the EHT collaboration to constrain the Kerr-Sen-like black hole parameters. We have observed that over a finite parameter space, black hole shadows satisfy the bound $\Delta C \leq 0.10$ both in the presence of plasma and in the absence of it.

Besides the result of the article [82] allows us to put put constrain on the value of $l$. We have found that the value of the parameter $l>-1$ and the upper bound of it is 0.621031 . So $l$ can take both positive and negative values.
Finally, we would like to mention that in the calculation of the rate of energy emission we did not consider the greybody factor. The mathematical formulation of it is much involved for any black hole and it is important if a quantitative estimation is attempted. Unfortunately, there is no available data to compare it with the real situation. A theoretical evaluation of it for this black hole may be done, which we are leaving for a separate study.

Data Availability Statement This manuscript has no associated data or the data will not be deposited. [Authors' comment: It is a theoretical paper with numerical calculation. All the calculated date are given in the article.]

Open Access This article is licensed under a Creative Commons Attribution 4.0 International License, which permits use, sharing, adaptation, distribution and reproduction in any medium or format, as long as you give appropriate credit to the original author(s) and the source, provide a link to the Creative Commons licence, and indicate if changes were made. The images or other third party material in this article are included in the article's Creative Commons licence, unless indicated otherwise in a credit line to the material. If material is not included in the article's Creative Commons licence and your intended use is not permitted by statutory regulation or exceeds the permitted use, you will need to obtain permission directly from the copyright holder. To view a copy of this licence, visit http://creativecomm ons.org/licenses/by/4.0/.

Funded by $\mathrm{SCOAP}^{3}$.

\section{References}

1. B.P. Abbott et al., Phys. Rev. Lett. 116, 061102 (2016)

2. K. Akiyama et al. (Event Horizon Telescope Collaboration), First M87 Event Horizon Telescope results. I. The shadow of the supermassive black hole. Astrophys. J. 875, L1 (2019)

3. K. Akiyama et al. (Event Horizon Telescope Collaboration), First M87 Event Horizon Telescope results. II. Array and instrumentation. Astrophys. J. 875, L2 (2019)

4. K. Akiyama et al. (Event Horizon Telescope Collaboration), First M87 Event Horizon Telescope results. III. Data processing and calibration. Astrophys. J. 875, L3 (2019)

5. K. Akiyama et al. (Event Horizon Telescope Collaboration), First M87 Event Horizon Telescope results. IV. Imaging the central supermassive black hole. Astrophys. J. 875, L4 (2019)

6. K. Akiyama et al. (Event Horizon Telescope Collaboration), First M87 Event Horizon Telescope results. V. Physical origin of the asymmetric ring. Astrophys. J. 875, L5 (2019)

7. K. Akiyama et al. (Event Horizon Telescope Collaboration), First M87 Event Horizon Telescope results. VI. The shadow and mass of the central black hole. Astrophys. J. 875, L6 (2019)

8. J.L. Synge, Mon. Not. R. Astron. Soc. 131, 463 (1966)

9. K. Hioki, K.I. Maeda, Phys. Rev. D 80, 024042 (2009)

10. L. Amarilla, E.F. Eiroa, G. Giribet, Phys. Rev. D 81, 124045 (2010)

11. F. Atamurotov, B. Ahmedovand, A. Abdujabbarov, Phys. Rev. D 88, 064004 (2013)

12. S.W. Wei, Y.X. Liu, J. Cosmol. Astropart. Phys. 11, 063 (2013)

13. A. Abdujabbarov, F. Atamurotov, Y. Kucukakca, B. Ahmedov, U. Camci, Astrophys. Space Sci. 344, 429 (2013)

14. F. Atamurotov, A. Abdujabbarov, B. Ahmedov, Astrophys. Space Sci. 348, 179 (2013)

15. U. Papnoi, F. Atamurotov, S.G. Ghosh, B. Ahmedov, Phys. Rev. D 90, 024073 (2014) 
16. A. Grenzebach, V. Perlick, C.I. Nammerzahl, Phys. Rev. D 89, 124004 (2014)

17. Z. Li, C. Bambi, J. Cosmol. Astropart. Phys. 2014, 041 (2014)

18. Z. Stuchlik, D. Charbulak, J. Schee, Eur. Phys. J. C78, 180 (2018)

19. M. Ghasemi-Nodehi, Z. Li, C. Bambi, Eur. Phys. J. C. 75 (2015)

20. A. Abdujabbarov, M. Amir, B. Ahmedov, S.G. Ghosh, Phys. Rev. D. 93, 104004 (2016)

21. F. Atamurotov, S.G. Ghosh, B. Ahmedov, Eur. Phys. J. C. 76 (2016)

22. G.S. Bisnovatyi-Kogan, O.Y. Tsupko, Phys. Rev. D 98, 084020 (2018)

23. R. Kumar, S.G. Ghosh, A. Wang, Phys. Rev. D 100, 124024 (2019)

24. S.W. Wei, Y.X. Liu, R.B. Mann, Phys. Rev. D 99, 041303 (2019)

25. M. Khodadi, A. Allahyari, S. Vagnozzi, D.F. Mota, JCAP 2009, $026(2020)$

26. M. Khodadi, A. Allahyari, S. Vagnozzi, D.F. Mota, JCAP 2002, $003(2020)$

27. D.O. Muhleman, I.D. Johnston, Phys. Rev. Lett. 17, 455 (1966)

28. D.O. Muhleman, R.D. Ekers, E.D. Fomalont, Phys. Rev. Lett. 24, 1377 (1970)

29. G.S. Bisnovatyi-Kogan, O.Y. Tsupko, Mon. Not. R. Astron. Soc. 404, $1790(2010)$

30. O.Y. Tsupko, G.S. Bisnovatyi-Kogan, Gravit. Cosmol. 18, 117 (2012)

31. O.Y. Tsupko, G.S. Bisnovatyi-Kogan, Gravit. Cosmol. 20, 220 (2014)

32. V. Morozova, B. Ahmedov, A. Tursunov, Astrophys. Space Sci. 346, 513 (2013)

33. G.S. Bisnovatyi-Kogan, O.Y. Tsupko, Universe 3, 1 (2017)

34. A. Abdujabbarov, B. Toshmatov, J. Schee, Z. Stuchlyk, B. Ahmedov, Int. J. Mod. Phys. D 26, 1741011 (2017)

35. A. Abdujabbarov, B. Ahmedov, N. Dadhich, F. Atamurotov, Phys. Rev. D 96, 084017 (2017)

36. C.A. Benavides-Gallego, A.A. Abdujabbarov, C. Bambi, Eur. Phys. J. C 78, 694 (2018)

37. V. Perlick, O.Y. Tsupko, G.S. Bisnovatyi-Kogan, Phys. Rev. D 92 , $104031(2015)$

38. F. Atamurotov, B. Ahmedovand, A. Abdujabbarov, Phys. Rev. D 92, 084005 (2015)

39. V. Perlick, OYu. Tsupko, Phys. Rev. D 95, 104003 (2017)

40. G.Z. Babarr, A.Z. Babar, F. Atamurotov, Eur. Phys. J. C 80, 761 (2020)

41. V.A. Kostelecky, S. Samuel, Phys. Rev. Lett. 63, 224 (1989)

42. V.A. Kostelecky, S. Samuel, Phys. Rev. D 39, 683 (1989)

43. V.A. Kostelecky, S. Samuel, Phys. Rev. D 40, 1886 (1989)

44. D. Colladay, V.A. Kostelecky, Phys. Rev. D 55, 6760 (1997)

45. D. Colladay, V.A. Kostelecky, Phys. Rev. D 55, 6760 (1997)

46. D. Colladay, V.A. Kostelecky, Phys. Rev. D 58, 116002 (1998)

47. V.A. Kostelecky, Phys. Rev. D 69, 105009 (2004)

48. R. Casana, A. Cavalcante, F.P. Poulis, E.B. Santos, Phys. Rev. D 97, 104001 (2018)

49. C. Ding, C. Liu, R. Casana, A. Cavalcante, Eur. Phys. J. C 80, 178 (2020)
50. S.K. Jha, A. Rahaman, Eur. Phys. J. C 81, 345 (2021)

51. A. Sen, Phys. Rev. Lett. 69, 1006 (1992)

52. P.V.P. Cunha, C.A.R. Herdeiro, E. Radu, Phys. Rev. Lett. 123(1), 011101 (2019)

53. D. Psaltis et al., Event Horizon Telescope. Phys. Rev. Lett. 125(14), $141104(2020)$

54. N. Ashish, S. Mohanty, A. Kumar, arXiv:2002.12786 (2020)

55. S.H. Volkel, E. Barausse, N. Franchini, A.E. Broderick, arXiv:2011.06812 (2020)

56. K. Glampedakis, G. Pappas, arXiv:2102.13573 (2021)

57. M. Afrin, R. Kumar, S.G. Ghosh, MNRAS 504, 5927 (2021)

58. S.U. Islam, S.G. Ghosh, arXiv:2102.08289

59. Z. Li, A. Ovgun, Phys. Rev. D 101, 024040 (2020)

60. J.L. Synge, Relativity: The General Theory. North-Holland, Amsterdam, 196

61. A. Abdujabbarov, B. Toshmatov, Z. Stuchlk, B. Ahmedov, arXiv: 1512.05206

62. A. Rogers, Mon. Not. R. Astron. Soc. 451, 17 (2015)

63. S. Dastan, R. Saffari, S. Soroushfar, arXiv: 1610.09477

64. S. Hawking, Commun. Math. Phys. 43, 199 (1975)

65. S.-W. Wei, Y.-X. Liu, J. Cosmol. Astropart. Phys. 11, 063 (2013)

66. U. Papnoi, F. Atamurotov, S.G. Ghosh, B. Ahmedov, Phys. Rev. D 90, $024073(2014)$

67. A. Abdujabbarov, M. Amir, B. Ahmedov, S.G. Ghosh, Phys. Rev. D 93, 104004 (2016)

68. B. Mashhoon, Phys. Rev. D 7, 2807 (1973)

69. C.W. Misner, K.S. Thorne, J.A. Wheeler, Gravitation (Freeman, San Francisco, 1973)

70. Y. Decanini, G. Esposito-Fareseand, A. Folacci, Phys. Rev. D 83, 044032 (2011)

71. O.Y. Tsupko, G.S. Bisnovatyi-Kogan, Gravit. Cosmol. 20, 220 (2014)

72. O.Y. Tsupko, G.S. Bisnovatyi-Kogan, Gravit. Cosmol. 18, 117 (2012)

73. O.Y. Tsupko, G.S. Bisnovatyi-Kogan, Gravit. Cosmol. 15, 184 (2009)

74. C.A. Benavides-Gallego, A.A. Abdujabbarov, C. Bambi, Eur. Phys. J. C 78, 694 (2018)

75. M. Fathi, M. Olivares, J.R. Villanueva, Eur. Phys. J C 81, 987 (2021)

76. G.V. Kraniotis, Gen. Relativ. Gravit. 46, 1818 (2014)

77. A. Abdujabbarov, B. Toshmatov, J. Schee, Z. Stuchlik, B. Ahmedov, Int. J. Mod. Phys. D 26, 1741011 (2017)

78. V.S. Morozova, B.J. Ahmedov, A.A. Tursunov, Astrophys. Space Sci. 346, 513 (2013)

79. T. Johannsen, D. Psaltis, Astrophys. J. 718, 446 (2010)

80. Cosimo Bambi, Katherine Freese, Sunny Vagnozzi, Luca Visinelli, Phys. Rev. D 100(4), 044057 (2019)

81. K. Gebhardt, J. Adams, D. Richstone, T.R. Lauer, S.M. Faber, K. Gultekin, J. Murphy, S. Tremaine, Astrophys. J. 729, 119 (2011)

82. R. Nemmen, Astrophys J. Lett. L26, 880 (2019) 